\title{
Analysis of the ASTM Round-Robin Test on Particle Size Distribution of Portland Cement: Phase II
}

Chiara F. Ferraris Vincent A. Hackley Ana Ivelisse Avilés Charles E. Buchanan, Jr. 



\section{Analysis of the ASTM Round-Robin Test on Particle Size Distribution of Portland Cement: Phase II}

Chiara F. Ferraris

Building and Fire Research Laboratory National Institute of Standards and Technology

Vincent A. Hackley Materials Science and Engineering Laboratory National Institute of Standards and Technology

Ana Ivelisse Avilés

Information Technology Laboratory National Institute of Standards and Technology

Charles E. Buchanan, Jr. Roan Industries, Inc. Bakersville, NC, (USA)

December 2002

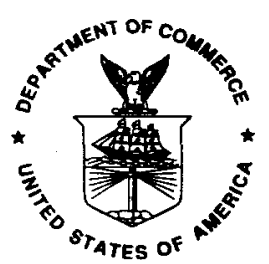

U.S. Department of Commerce Donald L. Evans, Secretary

Technology Administration Phillip J. Bond, Under Secretaryfor Technology 


\section{$\underline{\text { Abstract }}$}

A distribution of sizes or particle size distribution (PSD) is an essential property of cement powder. The only standard method to measure the PSD of cement, namely ASTM C115 [1] is limited in scope; this standard only describes a method for determining "fineness" with a lower size detection limit of $7.5 \mu \mathrm{m}$. As there is no standard procedure covering the whole range of cement PSD, the implementation of different measurement methods varies widely within the industry. A first report [2] was prepared to examine the methods used in the cement industry. The high variability of the data led to the necessity for further research.

ASTM committee C01.25.01 sponsored a second round-robin test to measure the PSD of cement. The aim of the current report is to analyze the data generated during that test and to summarize the various approaches available to measure the PSD of cement. The analysis of the data is conducted in two parts. In the first part, an attempt is made to establish a reference distribution using a standard cement powder (SRM 114p), improving the results already obtained from the first round-robin. This is followed by examination of the parameters and methodology used by the participants in order to initiate discussion on developing a standard test method for cement PSD to be submitted for ASTM consideration. The report provides all raw data collected during the round-robin tests, and the results of a statistical analysis of the collected data. 


\section{Acknowledgements}

The authors would like to thank the ASTM Task Group C01.25.01 for providing the opportunity for this round-robin. We would like to thank all participants of the round-robin (listed below by alphabetical order of the institution) for providing time and staff to perform the tests.

Also, we would like to thank Robin Haupt and the staff of the Cement and Concrete Reference Laboratory (CCRL), who were instrumental in providing the samples to the round-robin participants.

\section{Participants to the Round-Robin:}

William Phillips, Arizona Portland Cement

Kristen Freeman, Ash Grove Cement Technical Center

Ren Xu, Beckman Coulter

Rebecca Lara, CA. Portland Cement Co.

Luis Velasquez, Cementos Progreso, S.A.

Karen Ornelas, Cemex

Ella Shkolnik, Construction Technology Labs

Charlotte Lehmann, Dragon Products Co

Claus-Jochen Hacker, Dyckerhoff Zement

Thuy Ai Nguyen, Essroc Technology Center

Bjarne Osbaeck, F.L. Smidth A/S

Linda Lore, Florida Rock Industries

Muller-Pfeiffer, Forschumgsinstitut der Zementindustrie

Rita J. McKinley, Halliburton Energy Services

Dick Roush, Holcim

Michael Pohl, Horiba Instruments

Robin Cowdrey, Inland Cement Limited

Tom Griffiths, Lafarge

Lisa McEvoy, Harvi Sodhi and Daniel Decary, Lafarge Canada

Richard McCorkle, Lafarge North America

Jim Davis, Lehigh Cement

Jeffrey Hook, Lehigh Portland Cement

Tony Nutter and Ron Rajki, Lone Star Industries

Mike Bollinger and Henry Robinson, Lone Star Industries

Tom Gepford, Mitsubishi Cement Corp.

Boyd Clark, RJ Lee Group

Tany a Cable, ROAN Laboratories Inc.

Walter Pearce, St Marys Cement

John Falletta, St. Lawrence Cement, Inc.

Jesse Uribe, TXI Riverside Cement

Miquel Latorre, Uniland Cementera, S. A.

Vijay Gupta, W.R. Grace

Paolo Forni, W.R. Grace Italiana 


\section{TABLE OF CONTENTS}

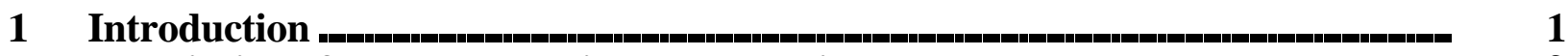

2 Description of methods used in the cement industry ________________ 2

$2.1 \quad$ Scanning Electron Microscopy (SEM) ....................................................................... 2

3 Analysis of data from the round-robin _._._. 4

3.1 Reference distribution using SRM 114p .................................................................. 5

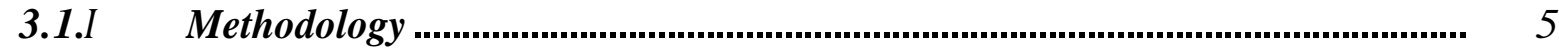

3.1.2 Determination of the reference distributionfor laser diffraction (Approach 2).....6

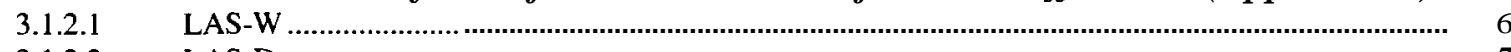

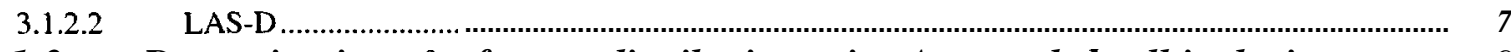

3.1.3 Determination of reference distribution using Approach 1: all-inclusive .............. 9

3.1.4 Correction procedure .................................................................................... 10

3.1.5 Summary .................................................................................................... 11

3.2 Analysis of methodologies ................................................................................... 16

3.2.I Laser diffraction with the specimen dispersed in a liquid (LAS-W) .................. 16

3.2.1.1 Summary of the participant's procedures for sample preparation ...........................................................16

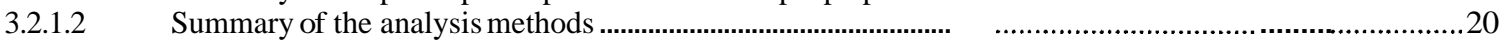

3.2.2 Laser diffraction with the specimen dispersed in air $(L A S-D)$......................... 23

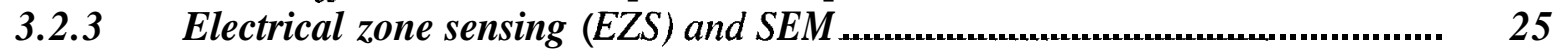

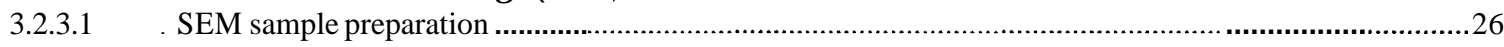

3.2.4 Summary ................................................................................................... 27

4 Recommendations and conclusions _._._.

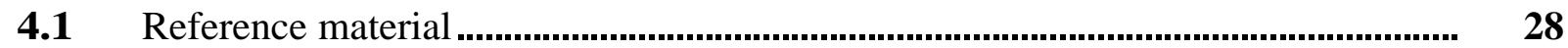

4.2 Standard method ........................................................................................... 28

$5 \quad$ References n........ 29

APPENDICES $\ldots \ldots \ldots \ldots \ldots \ldots \ldots \ldots \ldots \ldots \ldots \ldots \ldots \ldots \ldots \ldots \ldots \ldots \ldots \ldots \ldots \ldots \ldots \ldots \ldots \ldots . \quad$ A-31 


\section{LIST OF FIGURES}

Figure 1: Graphical comparison of the Bootstrap mean curves obtained in Table 1 to Table 3. For clarity. the standard deviations are not shown here. but can be found in the corresponding tables

Figure 2: Graphical comparison of the Bootstrap mean curves obtained in Table 4 to Table 6. For clarity, the standard deviations are not shown here, but can be found in the corresponding tables

Figure 3: Comparison of the mean LAS and the ESZ and SEM results

Figure 4: Graphical comparison between the distributions calculated from the one calculated

from only the LAS-W (Table 3) or LAS-D data (Table 6). For clarity, the standard

deviations are not shown here, but can be found in the corresponding tables.

Figure 5: Concentration of the dispersion in the cell for each participant. The values on top of the

bars represent values that are off the scale selected............................................................ 18

Figure 6: Distribution of the ultrasonication duration for all applicable participants................... 19

Figure 7: Distribution of the durations of the measurement ................................................... 20

Figure 8: Effectiveness of the duration of the sonication as shown by the dispersion of the

smaller particles ............................................................................................................. 21

Figure 9: Distribution of the optical model used with LAS-W ................................................ 23

Figure 10: Distribution of measurement durations by participant .......................................... 24

Figure 11:Relationship between the D 10 and the pressure used during the measurement in LAS-

D.

Figure 12: Distribution of the reported optical models used with LAS-D.................................. 


\section{LIST OF TABLES}

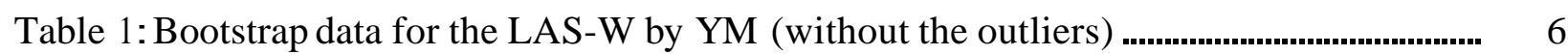

Table 2: Bootstrap data for the LAS-W by SPEC (without the outliers) ...................................... 7

Table 3: Bootstrap data for the LAS-W by SPEC and YM (without the outliers)......................... 7

Table 4: Bootstrap data for the LAS-D for YM (without the outliers) ........................................... 8

Table 5: Bootstrap data for the LAS-D for SPEC. No outliers were found ................................... 8

Table 6: Bootstrap data for the LAS-D by SPEC and YM (without the outliers)........................ 8

Table 7: Data from EZS (A15, 1773) and SEM (A1) ........................................................... 9

Table 8: Data from LAS-W by "your method" (YM) (see text). The results from the Bootstrap calculation are also shown. Data that are outside the $95 \%$ confidence limits are shown in bold and data that are more than $5 \%$ (absolute value) outside the $95 \%$ confidence limits are

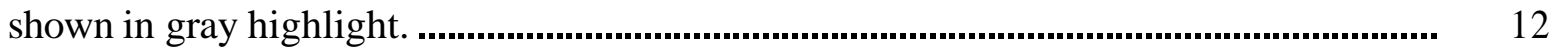

Table 9: Data from LAS-W by specification (SPEC) (see text). The results from the Bootstrap calculation are also shown. Data that are outside the $95 \%$ confidence limits are shown in bold and data that are more than $5 \%$ (absolute value) outside the $95 \%$ confidence limits are shown in gray highlight. .............................................................................................. 13

Table 10: Data from LAS-D for YM (see text). The results from the Bootstrap calculation are also shown. Data that are outside the $95 \%$ confidence limits are shown in bold and data that are more than $5 \%$ (absolute value) outside the $95 \%$ confidence limits are shown in gray highlight.

Table 11:Data from LAS-D for SPEC (see text). The results from the Bootstrap calculation are also shown. Data that are outside the $95 \%$ confidence limits are shown in bold and data that are more than $5 \%$ (absolute value) outside the $95 \%$ confidence limits are shown in

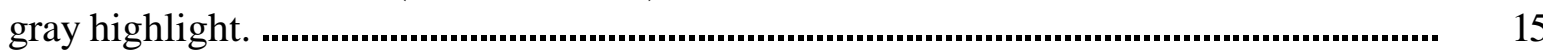

Table 12: Medium used for dispersing the cement. Total number of participants 26.................. 17

Table 13: Control of solids concentration in the measurement cell. The total number of

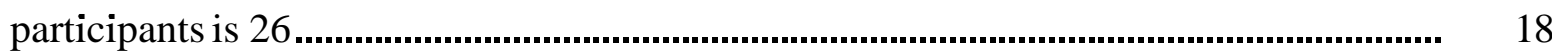

Table 14: The ultrasonication characteristics.......................................................................... 19

Table 15: Refractive indices used by the participants in LAS-W. If no value is shown, it signifies that no value was provided (not known) or used by that participant. The latter would include those using the Fraunhofer optical model. 


\section{Introduction}

This report summarizes the data obtained from the second cement particle size distribution (PSD) round-robin tests sponsored by ASTM committee C01.25.01. The first round-robin [2] involved 21 participants, while the second round-robin (reported here) involved 41 participants. Some participants took part in both round-robins. In both tests, there were two primary themes:

- Attempt to establish a reference cement particle size distribution using a standard cement (NIST-SRM 1 14p)

- Identify potential methods that could be used to draft a standard method

How to accurately measure the PSD of a cement powder is an important issue, because currently no standard or universally accepted method exists [3]. The cement PSD is essential for the complete characterization of a cement powder, as it is linked to its performance. The measurement of the cement PSD is rendered difficult by two main questions: 1) how to properly disperse the cement in a continuous medium, i.e., liquid or air; and 2) how to determine if the method yields the "correct" distribution (defining what is meant by "correct" is yet an additional issue in the context of method development and validation). Therefore, a reference material needs to be established, but also a methodology to disperse the cement in the medium needs to be drafted.

The aim of these two ASTM-sponsored round-robins is to address these questions. The reference material selected was SRM 114p, currently used primarily for Blaine measurements. A correct PSD of this cement needed to be established, and the approach taken was to establish a consensus curve. This pragmatic solution was required because establishing an analytically "correct" PSD, with our current understanding of how cement powders are structured and with currently available characterization techniques, was impractical and fundamentally unsound. The question of how to properly disperse the cement was addressed by conducting some detailed experiments at NIST, and by examining common industry practices as represented by participants in the second round-robin.

The two round-robins had some similarities and some differences. The same cement, SRM 114p, was used as a potential reference material, and various cements provided by CCRL were also included in the tests as was done in the first round-robin. However, the second round-robin requested that participants provide details describing their methodology and perform one set of measurements according to specified criteria with the goal of reducing user bias.

General information and the approach to data interpretation were described in the first report [2] and will not be repeated here unless necessary for the understanding of the present results. It is the intent of the authors to prepare a peer reviewed publication that would summarize the salient issues and combined results derived from the two round-robin tests. 


\section{Description of methods used in the cement industry'}

During the first round-robin the following methods were determined as being used for characterization of cement PSD:

\section{Laser Diffraction}

a. with the specimen dispersed in liquid (suspension-based)

b. with the specimen dispersed in air (aerosol-based)

2. Electrical Zone Sensing (Coulter Principle) (EZS)

3. Sedimentation

4. Sieving

5. Scanning Electron Microscopy (SEM)

The second round-robin included more participants (almost doubling, from 21 to 41 ), but the number of methods was reduced because sieving and sedimentation were not used by any other participants. The EZS method was used by only two participants; one participant was an instrument manufacturer, leading to the conclusion that EZS is not widely employed in the cement industry. SEM, considered a research-oriented method, was used by only one participant, also an instrument manufacturer.

Techniques listed above were detailed in the report prepared for the first round-robin [2], therefore, they will not be describe here. The only exception being the SEM method, which was significantly modified from the previous round-robin.

\subsection{Scanning Electron Microscopy (SEM)}

The SEM is an analytical tool that uses a focused beam of electrons to form magnified images. Under ideal conditions, the SEM is capable of producing images with a feature resolution at the nanometer $\left(10^{-9} \mathrm{~m}\right)$ level. In addition to image capabilities, a properly equipped SEM can provide information on the elemental composition of microscopic features. Information on the image and elemental characteristics of a sample are obtained through the interaction of the electron beam with the sample material, which produces various effects that can be monitored with suitable detectors. The resulting signals, which include secondary and backscattered electrons along with characteristic photoelectron X-rays, can be collected in synchronization with the position of the electron beam to provide detailed spatial and compositional information. Simply stated, secondary and backscattered electron signals provide image information, while Xrays are used to determine elemental composition (except for light elements such as $\boldsymbol{C}$ ).

The computer controlled SEM (CCSEM) can provide simultaneous measurement of individual particle size, shape (aspect ratio), and elemental composition by combining a scanning electron microscope (SEM), an X-ray analyzer (EDS), and a digital scan generator under computer

\footnotetext{
${ }^{\mathrm{I}}$ Commercial equipment, instruments, and materials mentioned in this report are identified to foster understanding. Such identification does not imply recommendation or endorsement by the National Institute of Standards and Technology (NIST), nor does it imply that the materials or equipment identified are necessarily the best available for the purpose.
} 
control. Use of the computer to control the analysis permits relatively large numbers of individual particles to be analyzed in a time efficient manner. During the CCSEM analysis, fields on the samples were analyzed "in order", i.e., the particles were detected on the support (see section 3.2.3.1 for details on the preparation) by moving the electron beam in discrete increments (" $x, y$ " pattern) across the sample and monitoring the resultant backscattered signal to determine when the electron beam was on a particle. The average, maximum, and minimum diameters were recorded during the analysis. Approximately 10000 to 11000 particles were analyzed from each sample. This included scanning the sample area at a magnification of $100 \mathrm{X}$ for 2500 particles greater than $10 \mathrm{pm}$. Then, 6000 particles from $1 \mu \mathrm{m}$ to $10 \mu \mathrm{m}$ in average diameter were analyzed at a magnification of $800 \mathrm{X}$ and finally 2500 particles were analyzed from 0.04 to $1 \mu \mathrm{m}$ in average diameter. Details on the method can be found in several publications $[4,5,6]$. Upon measurement of the particle size, the elemental composition of the particle was determined through collection of characteristic X-rays that were generated when the electron beam was on the particle. The elemental composition was neither requested nor is analyzed in this report, which is strictly related to the PSD. 


\section{Analysis of data from the round-robin}

ASTM committee C01.25.01 sponsored the second phase round-robin test to measure the PSD of cement. The participants were asked to use the PSD technique that they routinely employ. If their technique was based on laser diffraction (either wet/liquid dispersion or dry /aerosol), they were also asked to make measurements on SRM 114p using a preset method provided by NIST. The data requested from the participants included the cumulative PSD of three runs on each cement. Also, a detailed analysis report was to be returned with the data. All data were collected electronically. Forty-one organizations participated with the following methods represented:

- $\quad$ Laser diffraction wet (LAS-W)

- Laser diffraction dry (LAS-D)

- EZS

- SEM
25 participants

13 participants

2 participants

1 participant

As one organization provided two sets of data measured by LAS-W at different ultrasonication conditions, we really have 42 sets total including 26 sets in laser diffraction wet. The identity of each participant's organization remains confidential; therefore an alphanumeric code is used to represent participants in the data analysis. Each participant knows their individual code, but is unaware of the codes for the other participants.

Two portland cements provided by CCRL were included in the tests: 143 and 144 (the numbers were assigned by CCRL). The characteristics of these cements, as measured in the CCRL proficiency program, are given in Appendix A. The standard cement, SRM 114p, was also used to establish a reference PSD for cement. SRM 114p is routinely used to calibrate Blaine as well as other surface area measurements.

Appendix B summarizes in a tabular format all averaged PSD data received from the participants for CCRL cements 143 and 144. The data received for SRM 114p are shown in Table 8 to Table 11. In the remainder of this section, the data are analyzed first to establish the reference distribution using SRM 114p and then to provide a detailed examination of the methods used by each participant. Appendices $\mathrm{C}$ and D provide a copy of the type of information requested.

Three runs of the same powder sample were reported for each test material. An average curve was calculated using a simple arithmetic mean. Since participants may report different numbers of points or different size increments for the measured PSD, depending on the specific instrument and test parameters used, each data set was reduced to 15 sizes given in pm: $1,1.5$, $2,3,4,6,8,12,16,24,32,48,64,96$, and 128. This provides a more convenient basis for analysis and comparison. The reduction was made by simply combining the cumulative results reported by the participants. For instance, if data were reported for $0.1 \mathrm{pm}, 0.5 \mu \mathrm{m}$ and $1 \mathrm{pm}$, the sum of the cumulative percentage was reported for $1 \mathrm{pm}$. 


\subsection{Reference distribution using SRM 114p}

\subsubsection{Methodology}

As was done in the first round-robin analysis, results for SRM 114p were analyzed separately from the other cements with the objective of producing a reference material that instrument operators could use to "calibrate" their systems or at least to validate their methodology. In other words, the reference distribution of SRM 114p could be used to check that the PSD results obtained by a particular instrument fall within a defined margin of error, or it could be used to offset the measured values by a size-range-dependent factor in order to bring them within the acceptable margin of error. To achieve this goal, two approaches were considered:

1. Establish a single calibration curve that represents an average distribution for all methods inclusive (i.e., all-inclusive approach)

2. Establish a single calibration curve for each method, e.g., LAS-W or EZS (i.e., methodspecific approach)

Both approaches have advantages and disadvantages. In the first approach (all-inclusive), the calibration curve would be less precise (greater margin of error) due to propagation of uncertainties as a result of variations in the precision of different methods. On the other hand, the first approach is simpler and more convenient because all customers would use the same calibration curve. In the second approach (method-specific), the calibration should be more precise, because variations resulting from differences in measurement principle or precision between different techniques would be eliminated. As a disadvantage, several calibration curves would have to be established independently: one curve for each method.

There were 39 participants ( $93 \%$ of all participants) using the laser diffraction method. Of these, 26 (62\% of all participants) dispersed the specimen in a liquid (LAS-W) and 13 used a dry powder method (LAS-D). On the other hand, we had only one participant using SEM and two using EZS. Therefore, following the method-specific approach (Approach 2), we can determine only the calibration curve for LAS-W and LAS-D. Obviously, all 42 sets could be used if the allinclusive method (Approach 1) is followed, but the resulting curve would be heavily weighted by diffraction results.

To determine the curve that best represents the results, outliers should not be considered in the calculation of the mean curve. Therefore, a key issue is elimination of outliers from the calculation of the reference distribution. The method that was adopted here is based on the calculation of the mean and the two-sided $95 \%$ confidence limits using the bootstrap method (details on the bootstrap method are given in Appendix $\boldsymbol{C}$ of ref. [2]). The bootstrap method does not inherently provide the criteria needed to determine the outlier. Therefore, we selected the following criteria for elimination of outliers: if more than $\mathbf{2 7} \%$ of the points in a data set (four data points) are greater than $5 \%$ absolute value outside the confidence limits based on analysis of all data sets, then this data set is considered an outlier. The absolute value $5 \%$ is defined as the absolute difference between the measured value and the confidence limits. Once the outliers are determined, the mean and $95 \%$ confidence limits are recalculated excluding the outliers. This mean curve would be defined as the reference curve representing SRM $114 \mathrm{p}$. 
Therefore, in the following sections, various mean curves will be calculated depending on the two scenarios:

- All data

- Data from one method

\subsubsection{Determination of the reference distribution for laser diffraction (Approach 2)}

For the laser diffraction measurements, both wet and dry, two types of results were collected:

- Your method (YM): the participants were requested to use the method that they normally use and to describe it in detail

- Specifications (SPEC): the participants were requested to repeat the measurements using parameters specified by NIST (Appendix D).

Therefore, we will examine the two sets of data separately and then in combination after excluding the outliers. As a result of this process, three mean bootstrap curves with $95 \%$ confidence limits will be provided each for LAS-W and LAS-D. The issue is to decide which of these three distributions should be used as a reference for LAS-W and LAS-D. As shown in Figure 1 and Figure 2, the differences between the three distributions are not very large, and the combined distribution uses the largest number of data sets. Therefore, it could be argued that the combined PSD should be used as the reference curve. In this report, the combined distribution will be used to calculate the correction factors for the CCRL cements.

\subsubsection{LAS-W}

The calculated bootstrap mean and $95 \%$ confidence limits are shown in Table 8 for YM and in Table 9 for SPEC. Using the same criteria described in Section 3.1.1, the outliers are identified as

- A11S, A20 206,450,605 and $1251 \mathrm{~W}$ for YM.

- Al1S, A20, V6, 206, 450, 1483, and 2021 for SPEC

The bootstrap mean is then calculated without using the outliers. These results are shown in Table 1 for YM and Table 2 for SPEC. If all results obtained with LAS-W (i.e., both YM and SPEC) but excluding the outliers, are included, then the distribution is as shown in Table 3. A graphical comparison of the three distributions is given in Figure 1.

\begin{tabular}{|r|ccccccccccccccc|}
\hline $\begin{array}{r}\text { Size } \\
{[\mu \mathrm{m}]}\end{array}$ & 1 & 1.5 & 2 & 3 & 4 & 6 & 8 & 12 & 16 & 24 & 32 & 48 & 64 & 96 & 128 \\
\hline Mean & 5.0 & 7.9 & 11.1 & 16.3 & 20.5 & 27.9 & 34.1 & 45.8 & 54.7 & 69.7 & 80.4 & 92.6 & 97.1 & 99.4 & 99.9 \\
\hline Low & 3.5 & 6.4 & 9.6 & 14.8 & 19.0 & 26.5 & 32.8 & 43.9 & 52.7 & 68.0 & 78.8 & 91.5 & 96.4 & 99.0 & 99.7 \\
High & 6.3 & 9.5 & 12.5 & 17.8 & 22.1 & 29.4 & 35.5 & 47.8 & 56.6 & 71.6 & 82.1 & 93.7 & 97.9 & 99.7 & 100.0 \\
\hline
\end{tabular}




\begin{tabular}{|r|ccccccccccccccc|}
\hline $\begin{array}{r}\text { Size } \\
{[\mu \mathbf{m}]}\end{array}$ & 1 & 1.5 & 2 & 3 & 4 & 6 & 8 & 12 & 16 & 24 & 32 & 48 & 64 & 96 & 128 \\
\hline Mean & 6.0 & 9.5 & 13.7 & 19.0 & 23.4 & 30.7 & 37.3 & 49.0 & 58.0 & 72.5 & 83.4 & 94.3 & 98.3 & 99.7 & 100.0 \\
\hline Low & 3.9 & 7.2 & 11.6 & 16.7 & 21.1 & 28.3 & 34.9 & 45.7 & 55.0 & 69.8 & 81.3 & 93.0 & 97.5 & 99.5 & 99.9 \\
High & 8.3 & 12.1 & 15.9 & 21.3 & 25.7 & 33.3 & 39.7 & 52.1 & 61.2 & 75.1 & 85.3 & 95.5 & 98.9 & 99.9 & 100.0 \\
\hline
\end{tabular}

\begin{tabular}{|r|ccccccccccccccc|}
\hline $\begin{array}{r}\text { Size } \\
\text { [ } \boldsymbol{\mu m}]\end{array}$ & 1 & 1.5 & 2 & 3 & 4 & 6 & 8 & 12 & 16 & 24 & 32 & 48 & 64 & 96 & 128 \\
\hline Mean & 5.4 & 8.7 & 12.2 & 17.5 & 21.8 & 29.1 & 35.5 & 47.2 & 56.2 & 71.0 & 81.7 & 93.4 & 97.6 & 99.6 & 99.9 \\
\hline Low & 4.2 & 7.2 & 10.9 & 16.2 & 20.4 & 27.7 & 34.1 & 45.4 & 54.2 & 69.5 & 80.3 & 92.5 & 97.1 & 99.3 & 99.8 \\
High & 6.7 & 10.0 & 13.5 & 19.0 & 23.2 & 30.6 & 37.0 & 49.1 & 57.9 & 72.6 & 83.1 & 94.2 & 98.1 & 99.8 & 100.0 \\
\hline
\end{tabular}

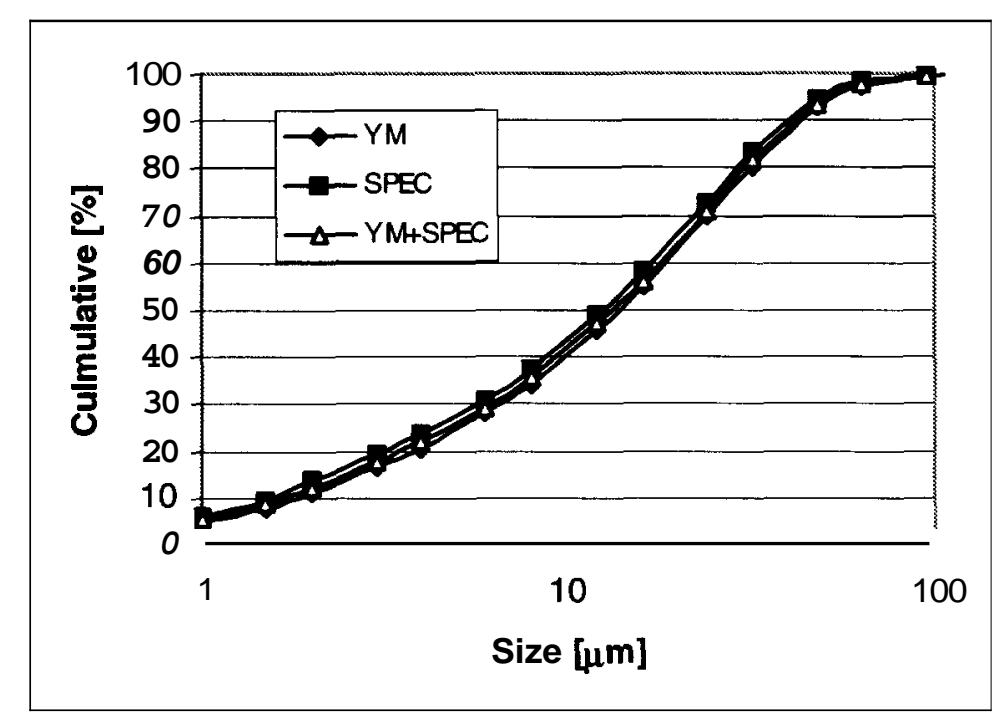

Figure 1: Graphical comparison of the Bootstrap mean curves obtained in Table 1 to Table 3. For clarity, the standard deviations are not shown here, but can be found in the corresponding tables.

\subsubsection{US - D}

The calculated bootstrap mean and $95 \%$ confidence limits are shown in Table $\mathbf{4}$ for $\mathbf{Y M}$ and in Table 5 for SPEC. Using the same criteria described in Section 3.1.1, the outliers are identified as

- A1 ID and A7 for YM.

- no outliers for SPEC 
The bootstrap mean is then calculated without using the outliers. This is shown in Table 4 for YM and in Table 5 for SPEC. If all results obtained with LAS-D (i.e., both YM and SPEC) excluding the outliers, are included, the distribution is as shown in Table 6. A graphical comparison of the three distributions is given in Figure 2.

\begin{tabular}{|r|ccccccccccccccc|}
\hline $\begin{array}{r}\text { Size } \\
{[\mu \mathrm{m}]}\end{array}$ & 1 & 1.5 & 2 & 3 & 4 & 6 & 8 & 12 & 16 & 24 & 32 & 48 & 64 & 96 & 128 \\
\hline Mean & 5.0 & 7.9 & 12.3 & 18.1 & 23.0 & 30.9 & 37.1 & 47.3 & 55.3 & 68.9 & 78.8 & 90.7 & 95.9 & 98.7 & 99.1 \\
\hline Low & 2.7 & 4.5 & 10.2 & 15.5 & 20.2 & 27.6 & 33.9 & 44.0 & 52.2 & 65.9 & 76.4 & 88.7 & 94.2 & 97.5 & 97.9 \\
High & 7.3 & 10.9 & 14.5 & 20.6 & 26.0 & 34.4 & 40.5 & 50.7 & 58.4 & 71.6 & 81.2 & 92.6 & 97.5 & 99.7 & 99.9 \\
\hline
\end{tabular}

\begin{tabular}{|r|ccccccccccccccc|}
\hline $\begin{array}{r}\text { Size } \\
{[\mu \mathrm{m}]}\end{array}$ & 1 & 1.5 & 2 & 3 & 4 & 6 & 8 & 12 & 16 & 24 & 32 & 48 & 64 & 96 & 128 \\
\hline Mean & 4.4 & 6.7 & 11.9 & 17.2 & 21.5 & 28.7 & 34.7 & 45.3 & 53.7 & 67.9 & 78.6 & 91.5 & 96.7 & 99.5 & 99.9 \\
\hline Low & 2.6 & 3.9 & 10.3 & 15.4 & 19.3 & 26.0 & 32.1 & 43.2 & 52.0 & 66.7 & 77.6 & 90.4 & 95.9 & 99.2 & 99.8 \\
High & 6.2 & 9.2 & 13.3 & 19.3 & 23.6 & 30.8 & 36.7 & 47.3 & 55.4 & 69.3 & 79.7 & 92.7 & 97.7 & 99.8 & 100.0 \\
\hline
\end{tabular}

Table 6: Bootstrap data for the LAS-D by SPEC and YM (without the outliers)

\begin{tabular}{|r|ccccccccccccccc|}
\hline $\begin{array}{r}\text { Size } \\
{[\mu \mathrm{m}]}\end{array}$ & 1 & 1.5 & 2 & 3 & 4 & 6 & 8 & 12 & 16 & 24 & 32 & 48 & 64 & 96 & 128 \\
\hline Mean & 4.7 & 7.3 & 12.1 & 17.7 & 22.3 & 29.9 & 35.9 & 46.4 & 54.6 & 68.4 & 78.7 & 91.1 & 96.3 & 99.1 & 99.5 \\
\hline Low & 3.3 & 5.4 & 10.6 & 16.1 & 20.3 & 27.8 & 33.9 & 44.4 & 52.8 & 66.9 & 77.3 & 89.9 & 95.3 & 98.3 & 98.8 \\
High & 6.1 & 9.5 & 13.4 & 19.3 & 24.2 & 32.0 & 38.1 & 48.4 & 56.7 & 70.1 & 80.2 & 92.2 & 97.3 & 99.6 & 100.0 \\
\hline
\end{tabular}




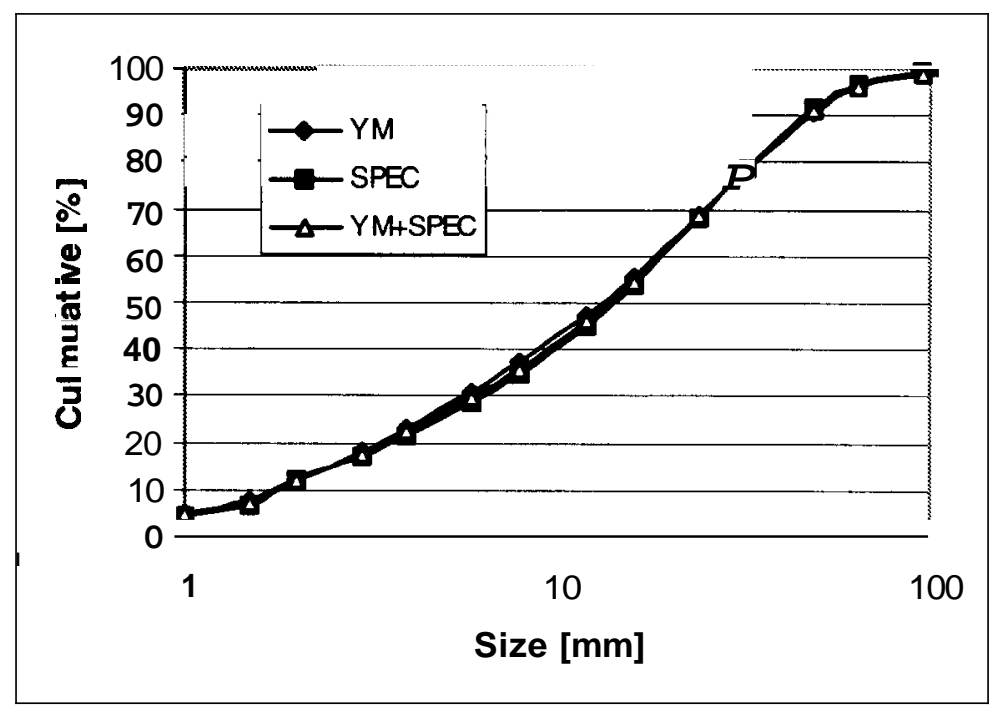

Figure 2: Graphical comparison of the Bootstrap mean curves obtained in Table 4 to Table 6. For clarity, the standard deviations are not shown here, but can be found in the corresponding tables

\subsubsection{Determination of reference distribution using Approach 1: all-inclusive}

Two other methods were used in this round-robin: EZS and SEM. As the data from LAS-W and LAS-D are already shown in previous tables and Sections, Table 7 will show only the data from the three participants using EZS and SEM. No mean could be calculated from these few tests. Also, if an overall mean using all methods (including LAS-W and LAS-D) was calculated, it could be argued that it is skewed toward the results obtained by the laser diffraction methods. As we cannot claim that the real PSD is the mean obtained using the laser diffraction methods, it is not statistically valid to calculate an overall mean PSD for all techniques. For information purpose only Figure 3 shows the data from ESZ and SEM compared with the mean value obtained by laser diffraction.

Table 7: Data from EZS (A15, 1773) and SEM (Al).

\begin{tabular}{|c|ccccccccccccccc|}
\hline $\begin{array}{r}\text { Size } \\
{[\mu \mathrm{m}]}\end{array}$ & 1 & 1.5 & 2 & 3 & 4 & 6 & 8 & 12 & 16 & 24 & 32 & 48 & 64 & 96 & 128 】 \\
\hline $\mathbf{A 1 5}$ & & & & 0.4 & 5.1 & 13.7 & 21.1 & 34.5 & 47.8 & 74.3 & 100 & 100 & 100 & 100 & 100 \\
\hline $\mathbf{1 7 7 3}$ & 0 & 0.3 & 0.6 & 1.5 & 2.9 & 6.2 & 10.1 & 18.8 & 28.4 & 48 & 63 & 82.6 & 90.3 & 100 & 100 \\
$\mathbf{A 1}$ & 1.4 & 3.7 & 6.9 & 13.8 & 19.9 & 34.5 & 45.9 & 64.2 & 75.4 & 89.6 & 96.9 & 100 & 100 & 100 & 100 \\
\hline
\end{tabular}




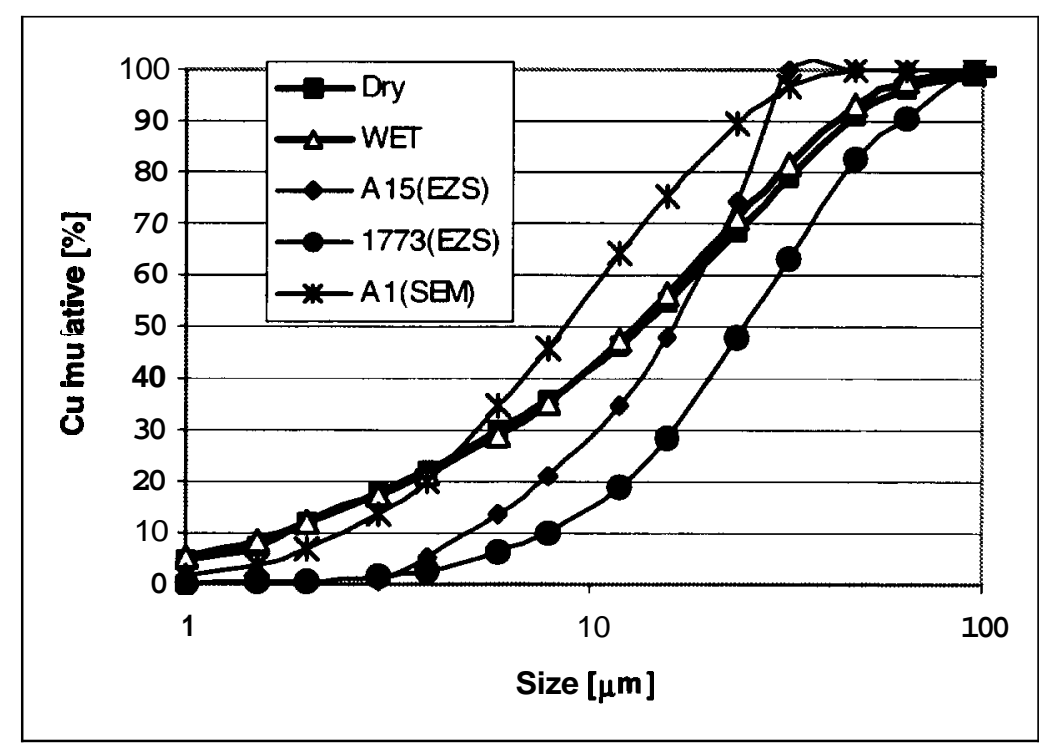

Figure 3: Comparison of the mean LAS and the ESZ and SEM results

\subsubsection{Correction procedure}

The purpose of a reference PSD based on an easily accessible reference material is twofold: 1) to verify the efficacy of the instrument or method being used, and 2) to correct measurement results by applying a set of correction factors. A methodology using the mean PSD curve to correct measured data obtained using various instruments would work in the following manner:

- Calculate the correction factor for each size, defined as the ratio between the measured value and the mean value as shown in Table 3 or Table 6 . The selection of the table depends on the method used.

- Multiply all measured data for unknown samples by this correction factor.

This procedure was applied to the two cements (CCRL \#143 and 144) used in this study, for all available data sets. In Appendix E, the corrected data and the correction factors are shown using the method-specific reference curve (Approach 2).

The ASTM committee was hoping that a single method and reference PSD could be used to correct all measurements. Unfortunately, in practice this proved more complicated. If the target measurement results themselves (not the calibration curve) contain outliers, (i.e., data points that are more than $5 \%$ absolute value outside the confidence limits obtained with the bootstrap method), the correction is not sufficient to bring the entire curve within the confidence limits of the calibration curve. This can be seen in Appendix E. On the other hand, if the data set lies completely within the confidence limits defined by the reference curve, the correction factor will reduce the spread of the data. Therefore, the reference SRM 114p could be used in two ways:

- To check that measurements are within the confidence limit range of the reference. This will allow the operator to determine if sample preparation problems or a malfunctioning instrument should be considered (Le., as a validation method). 
- To calibrate the instrument by correcting the results obtained using the reference cement, after the method has been validated.

\subsubsection{Summary}

Two approaches to determine the reference distribution for cement were examined. In Approach 1 all available data, without consideration of the measurement method, were included. In Approach 2, only the data generated with the LAS-W or LAS-D method were used. Only in Approach 2 could mean PSDs be generated using either LAS-W or LAS-D, and these mean distributions are shown in Figure 4. Though both curves appear to follow the same general form, there is some significant scatter apparent. The decision that needs to be made is which curve should be considered for assignment to a reference material. A discussion at the ASTM committee level could yield a consensus answer, but the authors propose that all available data (excluding outliers), from both Phase I and Phase II round-robins, should be included in the assignment of a reference curve for 114P. Two curves should be provided: 1) LAS-W, 2) LASD. An all inclusive curve (covering all techniques) would not be statistically correct due to the lack of data on methods not based on laser diffraction.

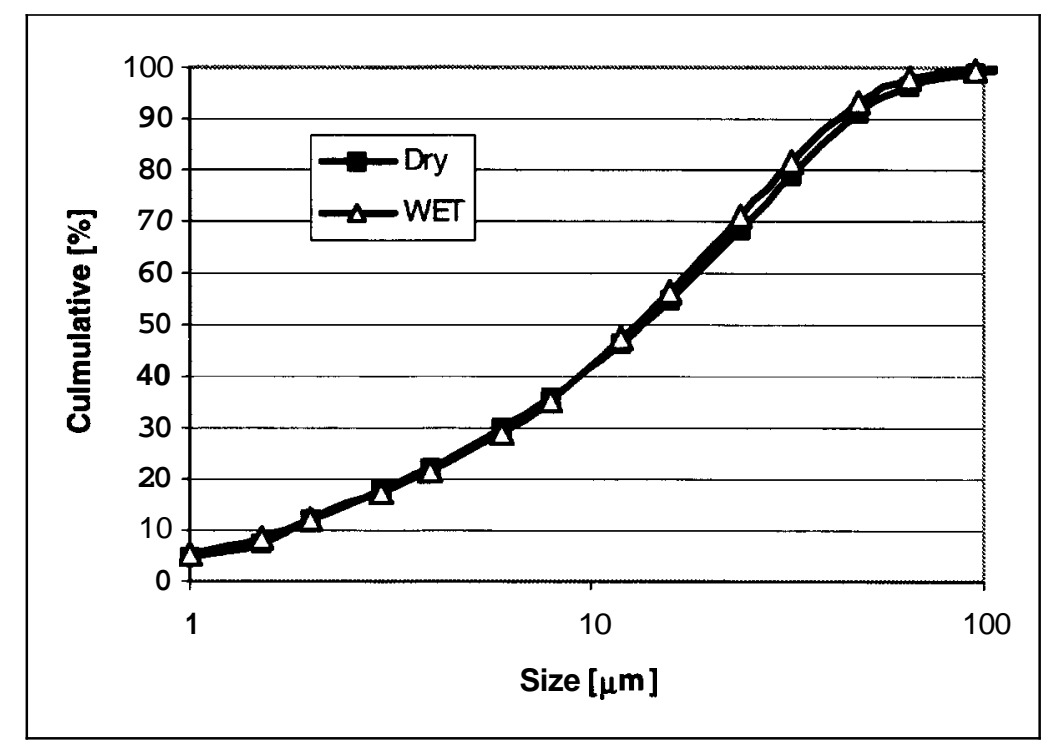

Figure 4: Graphical comparison between the distributions calculated from the one calculated from only the LAS-W (Table 3) or LAS-D data (Table 6). For clarity, the standard deviations are not shown here, but can be found in the corresponding tables. 


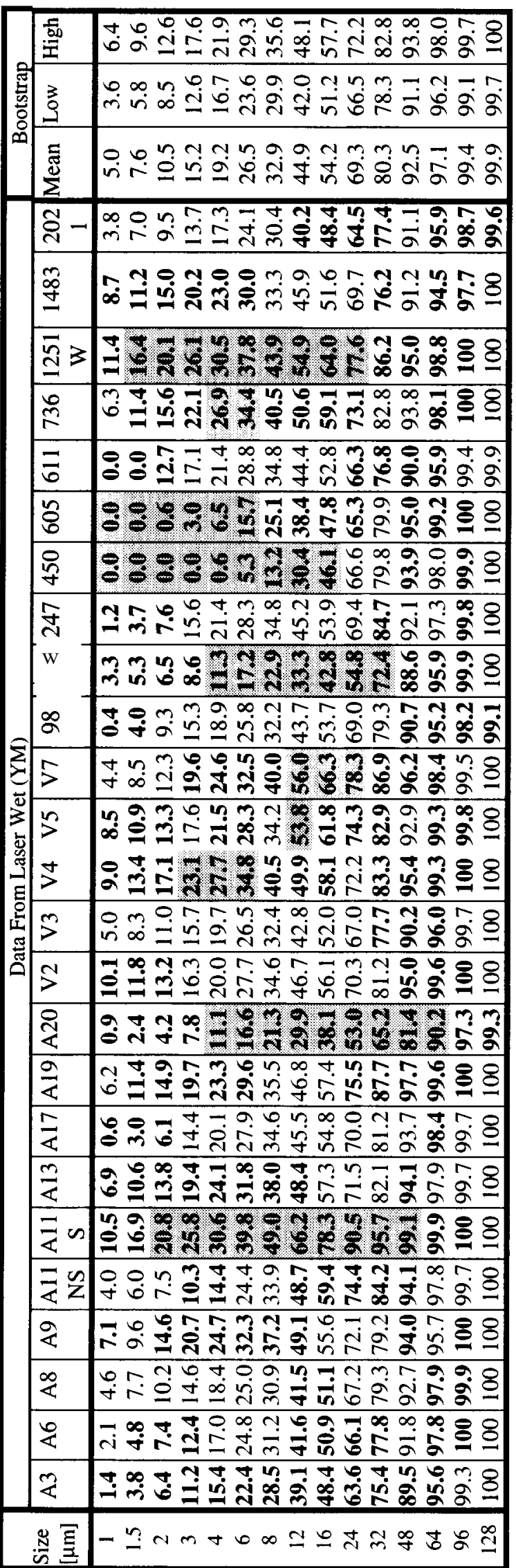




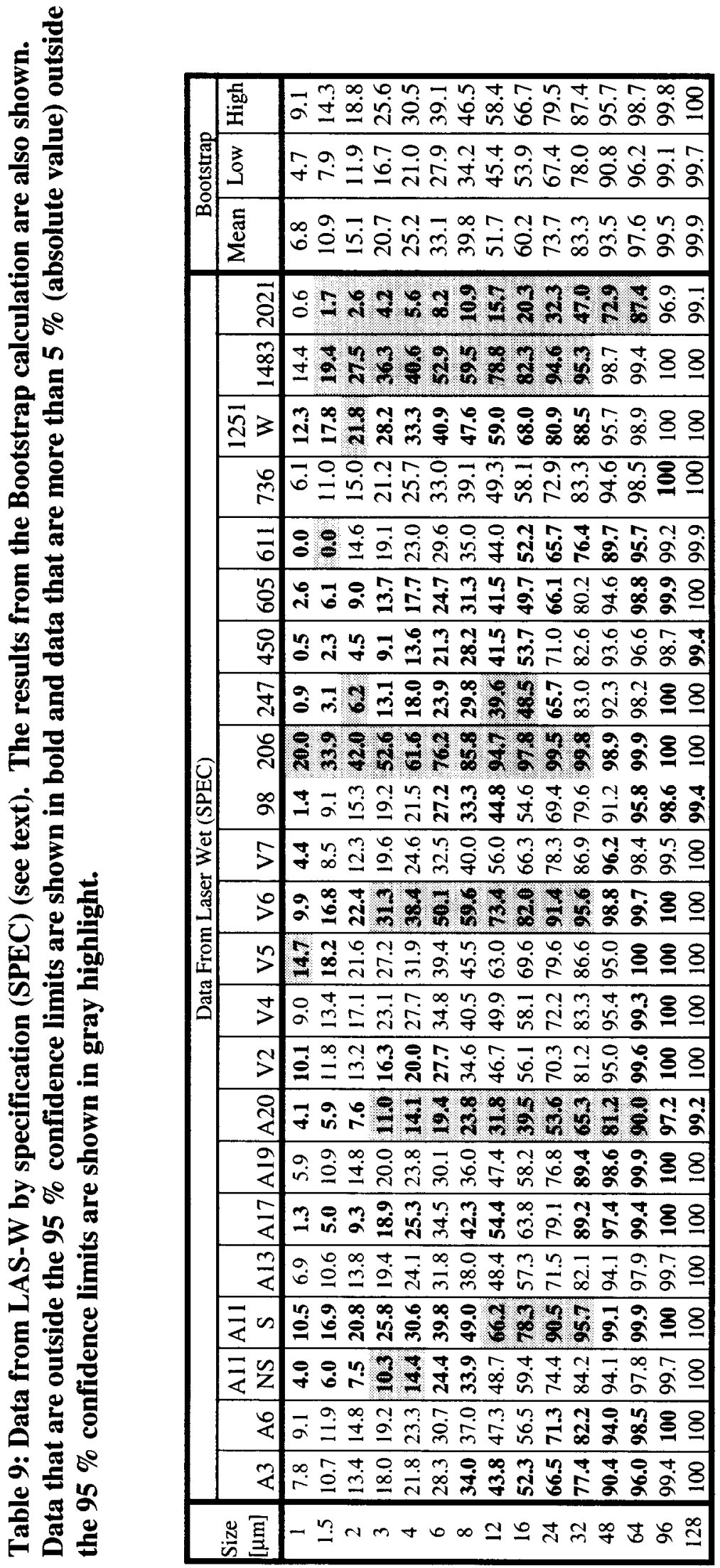




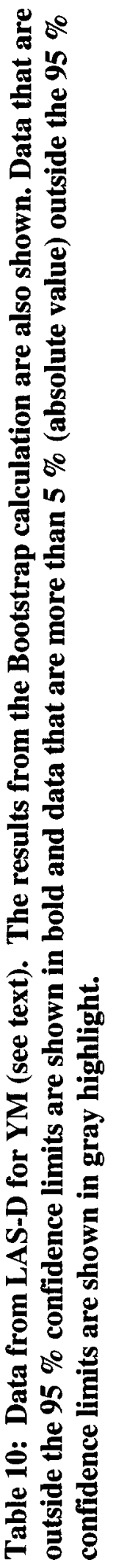

\begin{tabular}{|c|c|}
\hline .0.0 & 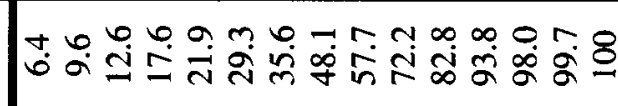 \\
\hline $0^{3}$ & نํํ \\
\hline 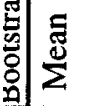 & 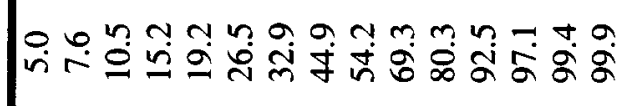 \\
\hline 吾 & 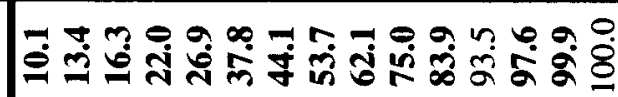 \\
\hline J & है용 \\
\hline 总 & 割 \\
\hline$\stackrel{m}{m}$ & 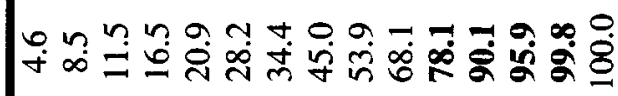 \\
\hline$\tilde{\pi}$ & 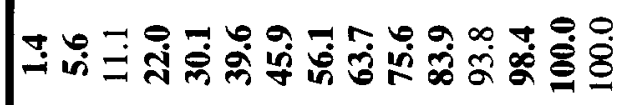 \\
\hline$\stackrel{\infty}{+}$ & 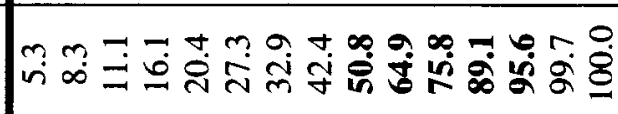 \\
\hline $\bar{\nu}$ & 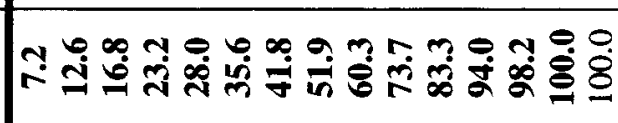 \\
\hline $\bar{y}$ & 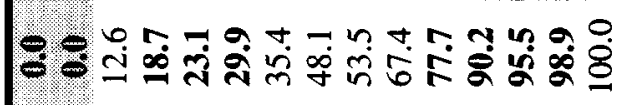 \\
\hline$\frac{0}{4}$ & 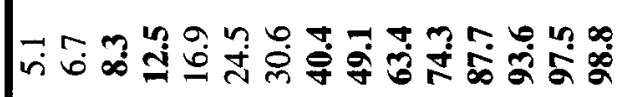 \\
\hline$\frac{\Delta}{4}$ & 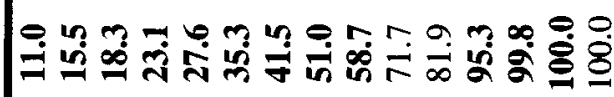 \\
\hline$\stackrel{\varrho}{4}$ & 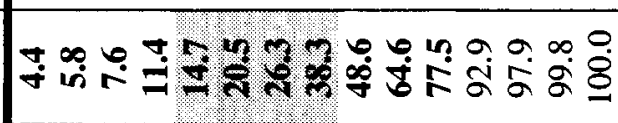 \\
\hline 4 & 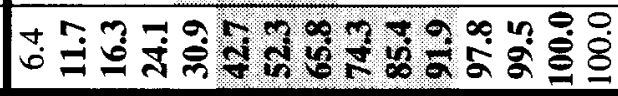 \\
\hline & \\
\hline
\end{tabular}




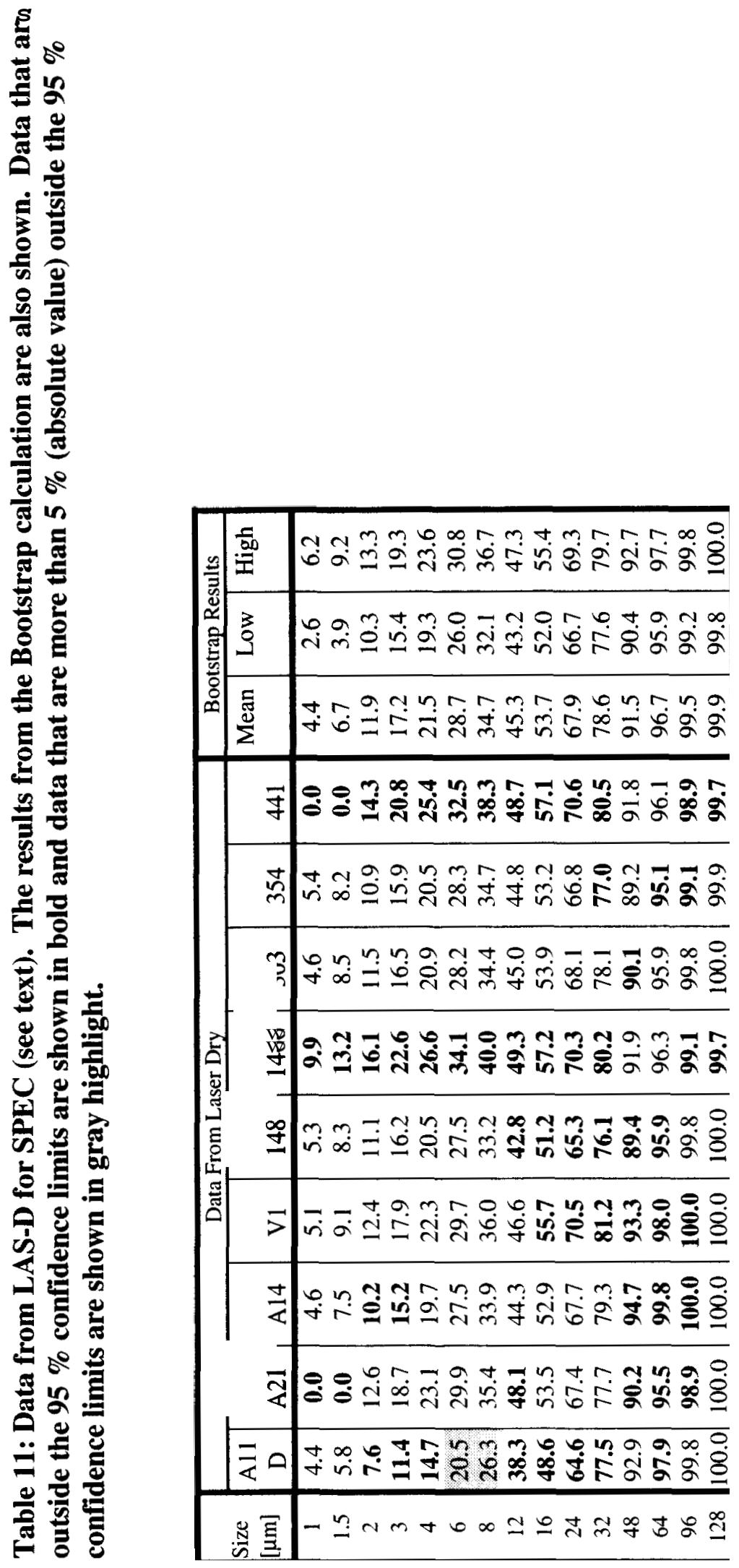




\subsection{Analysis of methodologies}

The scope of this round robin was also to compare the specimen preparation and measurement parameters used by industry for each method. To facilitate this comparison, participants were asked to provide specific detailed information about their in-house methods. A list of the requested information can be found in Appendix $C$. In this section we will examine the methods used and see if it is possible to determine a "best practice" that could eventually be presented to ASTM for approval as part of a standard test method. Since measurement parameters and sample preparation can be method-specific, each method will be examined separately.

\subsubsection{Laser diffraction with the specimen dispersed in a liquid (LAS-W)}

Participant-provided information concerning in-house methodology is divided into two areas: sample preparation and analysis. It is important to examine the responses with two goals in mind: 1) can a consensus procedure or procedures be established;2) can we identify key parameters that affect the results? Towards the first goal, an examination of the procedures used to obtain the outliers or the "best"distribution (defined as the closest to the mean bootstrap value; see Section 3.1) will provide a clue as to the best or consensus procedure. In the second case, a statistical analysis of the response information for specific procedures or parameters should help identify important aspects that need to be controlled or investigated more closely.

\subsubsection{Summary of the participant'sprocedures for sample preparation}

In the area of sample preparation, the following key information was requested where appropriate:

- Dispersion medium

- Concentration used and how dilution was achieved

- Surfactant

- Type and duration of ultrasonic treatment

Each of these issues should be clearly defined when a standard test is proposed to ASTM.

Table 12 shows a comparison of the reported medium used for dispersion of the cement powder in a liquid prior to and during analysis. Over $50 \%$ of the participants used isopropyl alcohol (IPA). There were only two participants who used a non-alcoholic medium (i.e., water-based). One of the participants (\#206) used water with no added surfactant and no ultrasonication. Participant \#450 used water, with no added surfactant, but ultrasonication of $20 \mathrm{~W}$ for $60 \mathrm{~s}$.

Previously (see Section 3.1.2), results \#206 and \#450 were both identified as outliers by the statistical analysis. Given the propensity for reaction of cement powder with water, this is not a unexpected result. It is possible that the PSD could change during the course of the measurement due to the hydration of cement, or that the reactive cement particles cling to the optical cell walls and thereby influence the precision and accuracy of the results. 


\begin{tabular}{|l|cc|}
\hline Medium & $\begin{array}{c}\text { Number of } \\
\text { participants }\end{array}$ & $\begin{array}{c}\text { Percentage of } \\
\text { total [\%] }\end{array}$ \\
\hline Ethanol & $\mathrm{a}$ & 31 \\
IPA & 14 & $\mathbf{5 4}$ \\
Methanol & 2 & 8 \\
Water & 2 & 8 \\
\hline
\end{tabular}

Examination of the correlation between the type of medium used and whether the data set is an outlier shows that:

- Data sets which used water are outliers.

- On the outlier list, there are three tests performed using IPA and one using ethanol.

From these observations, it could be inferred that water is not a suitable medium as those sets underestimate the finest fraction. Between ethanol and P A the number of outliers is proportional to the number of participants using those mediums. Therefore, no conclusions could be drawn on whether one of the alcohols was better than the another.

The second issue concerns the concentration of cement in the measuring cell and the dispersion method. This information is paramount because it can affect the capability to correctly disperse the cement and, therefore, could lead to a bias or increased variability in the measurements. It is clear from Table 13 that the vast majority of participants prepared their cement powder suspensions in one step (i.e., without dilution from a stock concentrate). In some cases a known amount of cement was added, while in other cases the addition amount was varied to achieve a certain optical obscuration level in the cell. The optimal percentage obscuration range was predetermined by the manufacturer of the device. As can be seen in Figure 5, the concentration varies widely and is reported explicitly by only 12 participants out of 26 who used liquid dispersion. It could be concluded from these results that the most common practice is to adjust concentration based on obscuration. It might therefore be difficult to prescribe a fixed solids concentration for a standard test method, since different instruments may require different obscuration levels. An alternative route would be to specify the solids content for a stock concentrate, which would be used to control sample dispersion properties. The stock could then be diluted as needed to obtain the optimal obscuration level for a particular instrument. Existing standards outside the U.S. should also be closely examined to determine the best method.

Only one participant (\#736) used a surfactant during sample preparation. The medium used in this case was ethanol and the surfactant was $\mathrm{SrCl}_{2}$ at a dose of $0.06 \mathrm{~g} / \mathrm{L}$. No conclusions can be drawn from this lone test. 
Table 13: Control of solids concentration in the measurement cell. The total number of participants is 26.

\begin{tabular}{|c|cc|}
\hline Dil $\begin{array}{c}\text { from } \\
\text { Stock } \\
\text { Y/N }\end{array}$ & Number of & Percentage \\
Norticipants & of total [\%] \\
\hline No & 19 & 73 \\
Yes & 6 & 23 \\
unknown & 1 & 4 \\
\hline
\end{tabular}

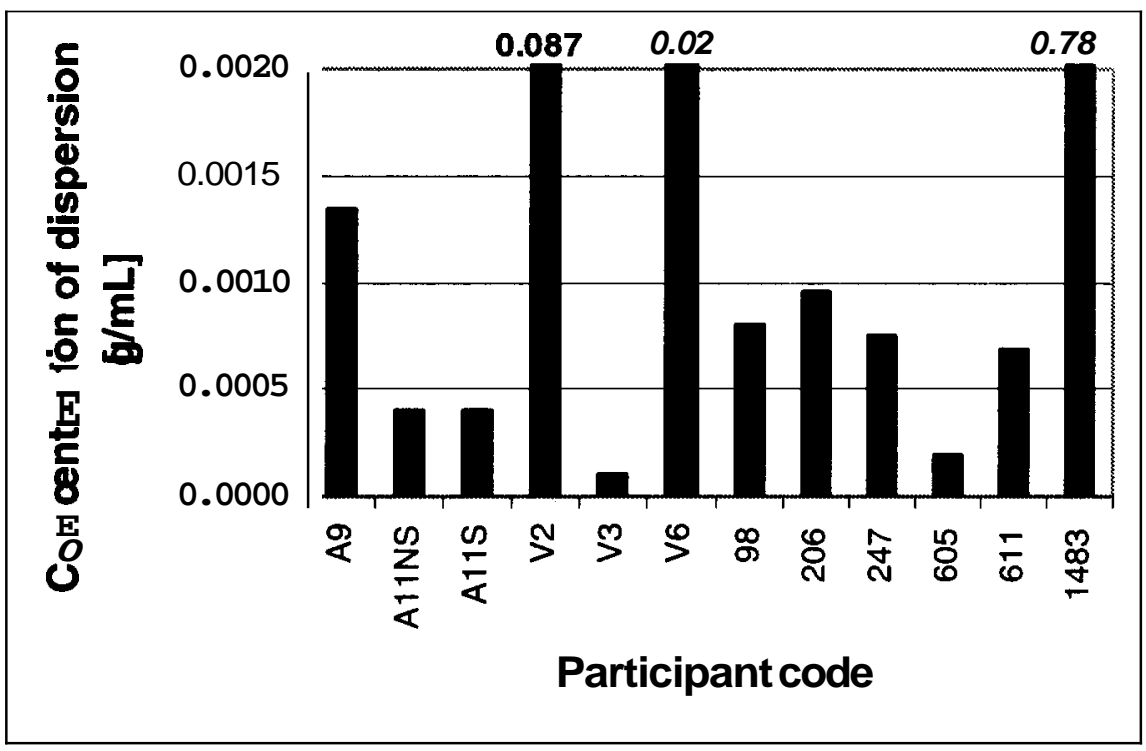

Figure 5: Concentration of the dispersion in the cell for each participant. The values on top of the bars represent values that are off the scale selected.

Another factor is how the dispersion was further prepared by using ultrasonication. Analysis results show that $69 \%$ employed ultrasonic treatment to disperse cement suspensions prior to measurement. Of these, $63 \%$ used the on-line ultrasonication provided by the instrument, while the remainder used an externally applied ultrasonic treatment prior to the introduction of the sample to the device. One participant (V2) reported using both external and in-line ultrasonication.

The power and the duration of the ultrasonication should be compared to determine the best procedure for an ASTM standard. Unfortunately, the power cannot be clearly compared because values are not always reported in fundamental units (i.e. Watts versus a relative \% scale) nor is output power always clearly defined with respect to the ultrasonic geometry and sample volume. In some cases the frequency is reported and in others it is not. Table 14 summarizes the available information. On the other hand, the duration is always reported and this is shown in Figure 6. The values range from $10 \mathrm{~s}$ to $300 \mathrm{~s}$, with a median value of $60 \mathrm{~s}$. It does not seem that there is a correlation between the duration and whether the distribution contains outliers. Further studies to 
determine the impact of ultrasonic treatment duration and power output on dispersion of cement in alcoholic media are ongoing at NIST. Preliminary results indicate that after an initial treatment duration $(<120 \mathrm{~s})$, further ultrasonic treatment provides no additional particle dispersion in alcoholic media.

Table 14: The ultrasonication characteristics

\begin{tabular}{|c|ccc|}
\hline $\begin{array}{c}\text { Participant } \\
\text { Code }\end{array}$ & Power & $\begin{array}{c}\text { Duration } \\
{[\mathrm{s}]}\end{array}$ & Type \\
\hline A6 & $7 \mathrm{~W}$ & 240 & on-line \\
A8 & $20 \mathrm{Khz}$ & 90 & on-line \\
A11S & $300 \mathrm{~W}$ & 90 & external \\
A13 & $125 \mathrm{~W}$ & 60 & external \\
A19 & N/A & N/A & ion-line \\
A20 & 13.1 On & 25 & external \\
& dial & & \\
V2 & High & 15 & external/on- \\
& & & line \\
V3 & $100 \%$ & 40 & on-line \\
V4 & $50 \mathrm{~W}$ & 60 & on-line \\
V5 & $50 \%$ & 30 & external \\
V7 & $40 \mathrm{~W}$ & 60 & external \\
98 & $40 \mathrm{~W}$ & 120 & on-line \\
247 & $80 \mathrm{~W}$ & 60 & external \\
605 & $40 \mathrm{~W}$ & 300 & on-line \\
736 & & 60 & on-line \\
1251 & & 60 & on-line \\
1483 & $20 \mathrm{Khz}$ & 120 & on-line \\
2021 & $25 \mathrm{~W}$ & 10 & on-line \\
\hline
\end{tabular}

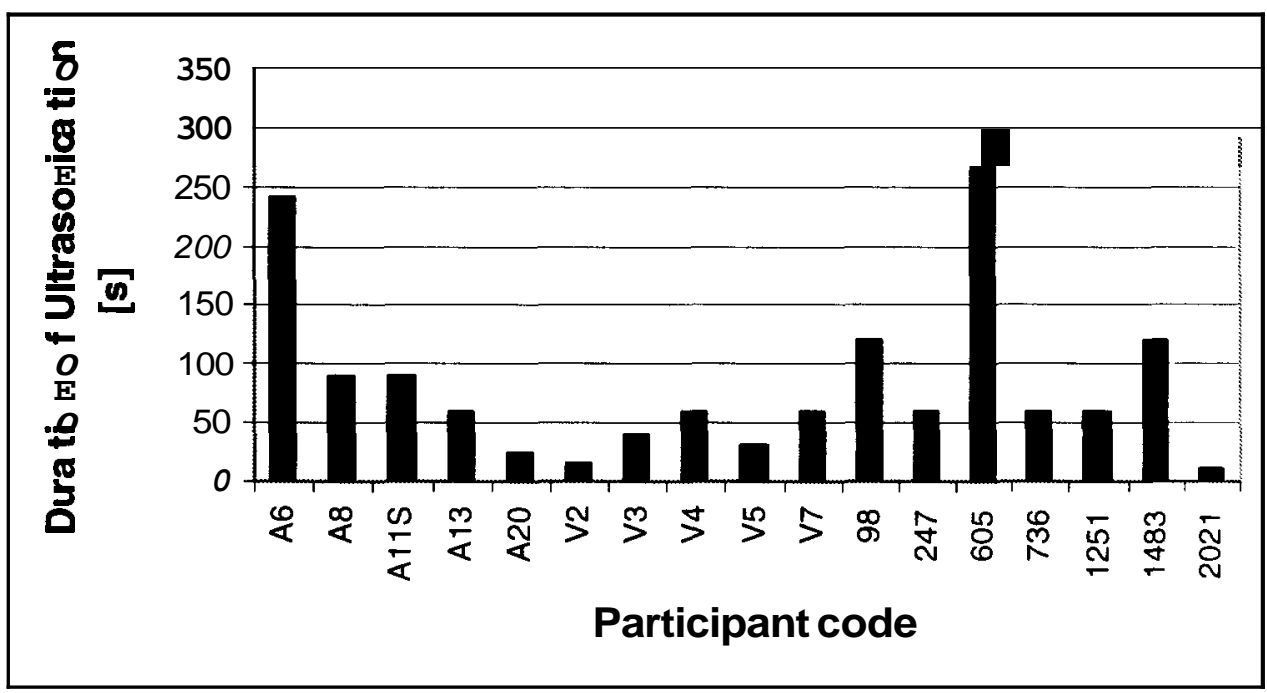

Figure 6: Distribution of the ultrasonication duration for all applicable participants. 


\subsubsection{Summary of the analysis methods}

There were three specifications requested from the participants with respect to the analysis step:

- Duration of the measurements

- Model used to interpret the results: Mie, Fraunhofer or both

- If Mie, complex refractive index used (real and imaginary) for the cement and the medium

The duration of the measurements varied from $4 \mathrm{~s}$ to $120 \mathrm{~s}$. This is a wide range that seems to depend on the particular commercial device used. Figure 7 shows the distribution of measurement durations obtained. It is clear that the majority of measurements last $\mathbf{6 0} s$ or less. Figure 8 shows the relationship between the sizes of the lower $10 \%\left(D_{10}\right)$ of the particles as a function of the duration of the sonication. If the sonication was effective, the sizes should be smaller with the higher sonication. It is clear that there is no correlation. This seems to imply that other factors influence the dispersion of the particles.

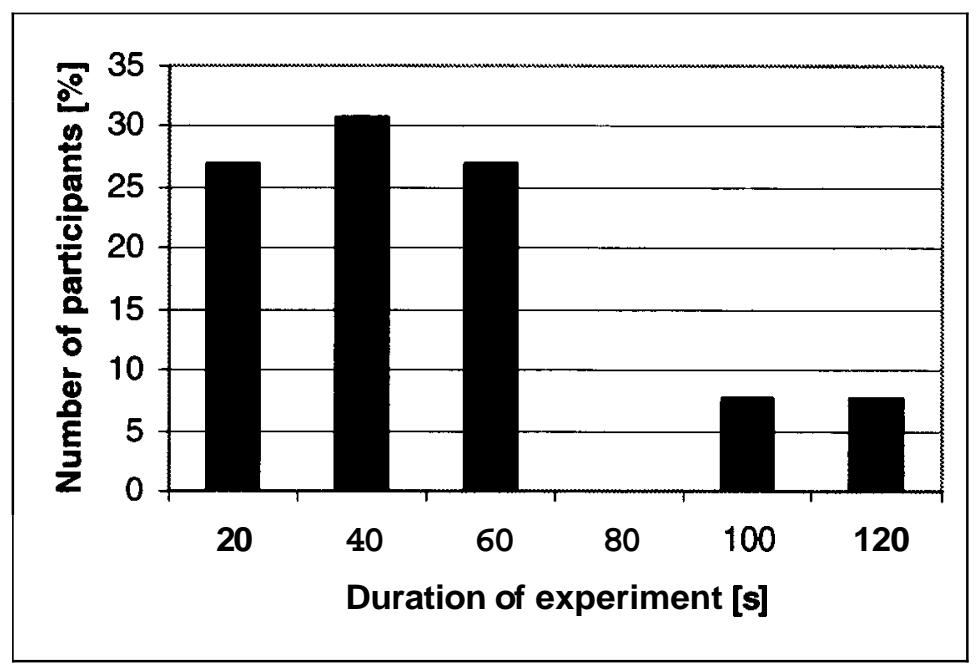

Figure 7: Distribution of the durations of the measurement. 


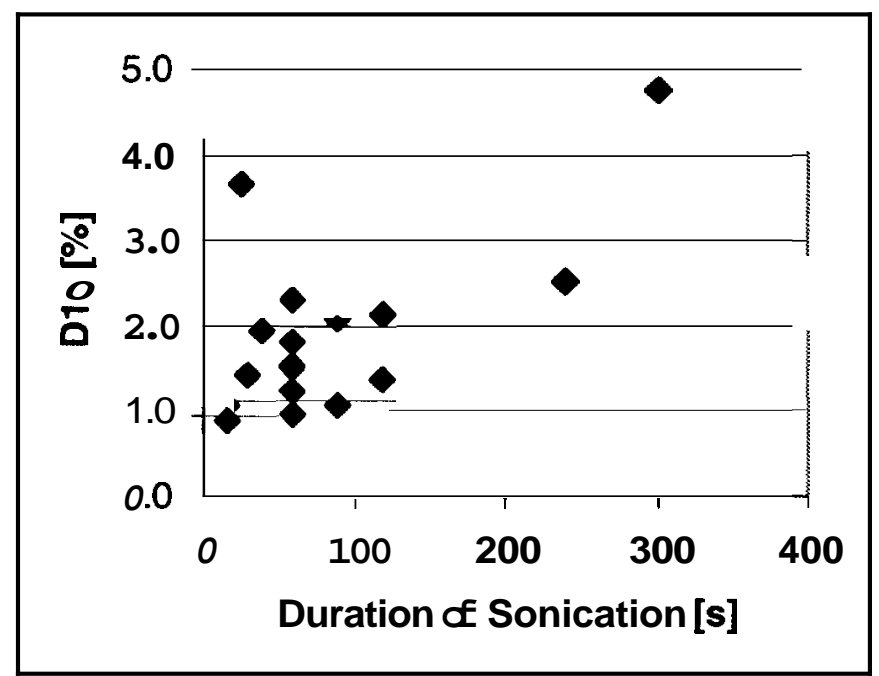

Figure 8: Effectiveness of the duration of the sonication as shown by the dispersion of the smaller particles.

The refractive index is critical if the Mie optical model is used to interpret the data and obtain the PSD of the cement. Table 15 shows the indices used by the participants reporting Mie results. The selection of the real refractive index of the powder varies from 1.23 to 1.88 . But if we exclude the single value at 1.23 (participant \#A19), the minimum value is 1.6 and the range is significantly narrowed. The median value is 1.725 , if 1.23 is excluded. Most participants (64\%) used 0.1 for the complex refractive index of cement. Other values reported were 0.01 (27\% of the participants) and 1.5 by a single participant (\#A19). A consensus value for the real and complex indices would yield 1.73 and 0.1, respectively. It should be noted that participant \#A19, while using refractive index values far from the mean, nevertheless was not determined to be an outlier in the statistical analysis presented in Section 3.1.2. Sources for the refractive indices reported by the participants were not requested and were not revealed. Since these values can vary with powder composition, it is an interesting observation that each participant apparently selected a single set of values and applied them to all cements irregardless of composition. If some consideration was given to the compositional variations during the selection process, it was not possible to determine this from the present study. The influence of variations in the real and imaginary components on the apparent PSD for cement is the subject of an on-going NIST investigation. 
Table 15: Refractive indices used by the participants in LAS-W. If no value is shown, it signifies that no value was provided (not known) or used by that participant. The latter would include those using the Fraunhofer optical model.

\begin{tabular}{|c|c|c|}
\hline \multirow{2}{*}{$\begin{array}{c}\text { Participanl } \\
\text { Code }\end{array}$} & \multicolumn{2}{|c|}{ Refractive index of powder } \\
\cline { 2 - 3 } Real & Imaginary \\
\hline A3 & 1.729 & $\mathbf{0 . 0 1}$ \\
A6 & 1.729 & $\mathbf{0 . 0 1}$ \\
A8 & 1.729 & 0.10 \\
A1 1 & 1.700 & 0.10 \\
A13 & 1.700 & 0.10 \\
A19 & 1.230 & 1.50 \\
A20 & 1.725 & 0.01 \\
v 3 & 1.680 & 0.10 \\
v 5 & 1.680 & 0.10 \\
V6 & 1.700 & 0.10 \\
98 & 1.810 & \\
206 & 1.680 & 0.10 \\
450 & 1.880 & \\
605 & 1.800 & \\
611 & 1.729 & \\
2021 & 1.600 & \\
\hline
\end{tabular}

There are generally two optical models for interpreting angle-dependent scattering by particles: Fraunhofer and Mie. Only the second one requires the refractive indices to be specified. According to ISO 13320-1 [7], the Fraunhofer model works well for particle sizes $>50$ pm. For particle sizes $<50 \mu \mathrm{m}$, the Mie model is preferred if a reasonable estimate of the refractive indices are available. In the intermediate range from about $1 \mu \mathrm{m}$ to $50 \mu \mathrm{m}$, the appropriateness of the choice of optical model will depend on whether the relative refractive indices (ratio of particle to medium) are high or low, and thus the decision is more complicated. In the submicrometer range, the Fraunhofer model is not applicable. The availability of different optical methods on a particular commercial instrument may also be a limiting factor for some users. Figure 9 shows the distribution of the methods as reported by the participants. It is clear that $80 \%$ of the participants use either Fraunhofer, Mie or both. It is surprising that as many as $16 \%$ of the participants seem unaware of which optical model they are using to analyze their data.

A standardized test method would have to account for the possibility that either the Fraunhofer or the Mie model might not be available to every instrument user. An ASTM standard should also recommend refractive indices to be used for certain types of cement, or, alternatively, a method for estimating these values based on the known composition of the powder. Further studies to establish the influence of the model choice and model parameters are underway at NIST. 


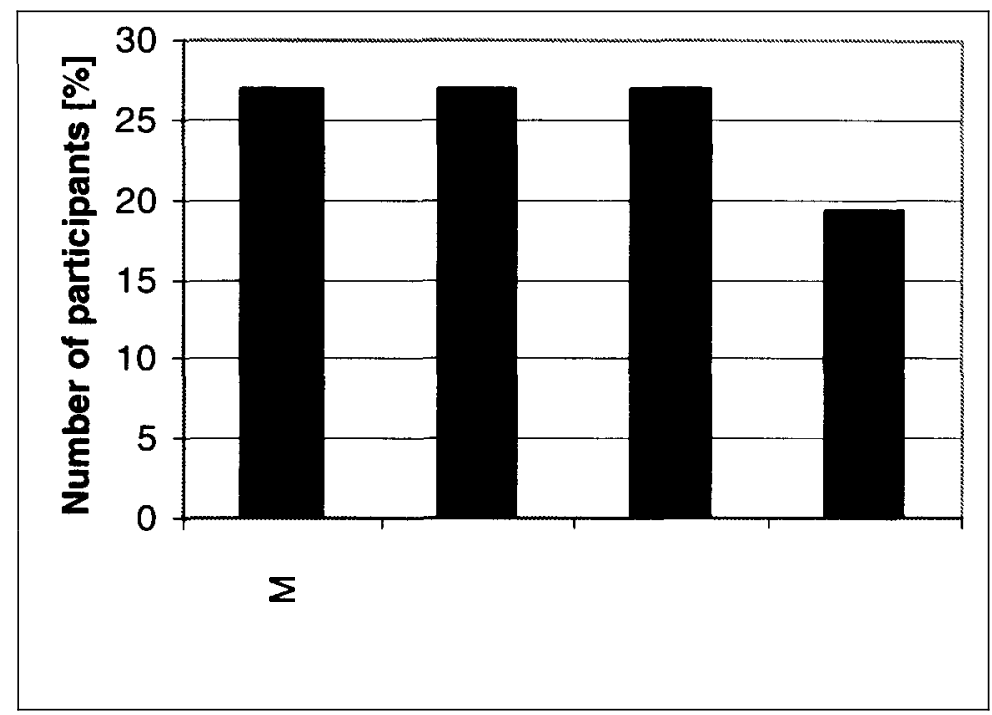

Figure 9: Distribution of the optical model used with LAS-W.

\subsubsection{Laser diffraction with the specimen dispersed in air (LAS-D)}

The sample preparation issue is greatly simplified in the case of LAS-D, since powders are introduced to the measurement device in dry form with dispersion provided internally by the instrument. Available aerosol dispersion methods for commercial LAS-D instruments are based on the use of compressed air, vacuum, or both in combination. In addition, each instrument company incorporates its own proprietary sample delivery and dispersion system, which might include, for instance, use of vibration, a venturi, or other mechanical devices. There were 13 participants who used LAS-D: $85 \%$ of those used systems based on compressed air, one used a vacuum based system (\#A14), and one used a system incorporating both compressed air and vacuum (\#441). One should keep in mind that this information was reported by the participant, and is not necessarily an accurate and complete assessment of the instrument's actual specifications or capabilities. Reporting bias or user ignorance may therefore impact these numbers somewhat. Another potentially significant measurement parameter, the duration of the measurements as reported, varied from $4 \mathrm{~s}$ to $130 \mathrm{~s}$. The median value was $15 \mathrm{~s}$ (Figure 10).

The pressure used during the measurement when compressed air was used varied from 1 bar to 4 bar. This is generally the maximum pressure that is available by a LAS-D. As shown in Figure 11 there is no correlation between the diameter of the smaller fraction of the particles and pressure used. 


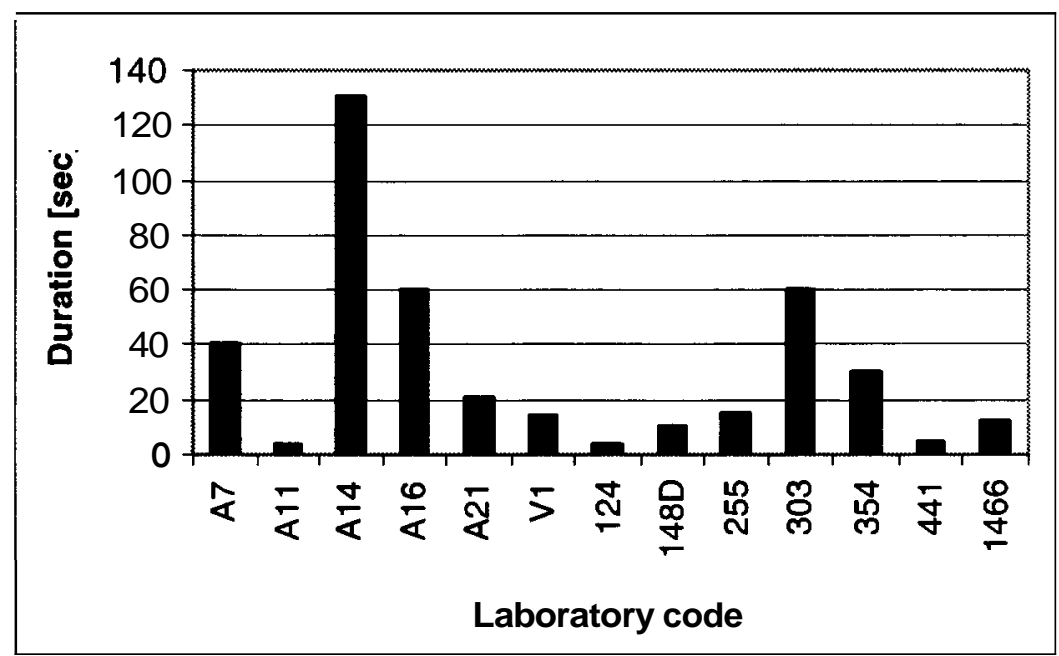

Figure 10: Distribution of measurement durations by participant

Like LAS-W, LAS-D requires the use of an appropriate optical model and, where appropriate, the selection of appropriate optical constants. Figure 12 shows the frequency in the reported optical model used for PSD analysis. The majority reported using the Fraunhofer model $(\approx 45 \%)$. The category "other" includes those not reporting a specific model or providing information that could not be clearly identified with either Fraunhofer or Mie. The variation in the refractive indices reported is very small for LAS-D, with only 8 participants reporting values. This is not surprising, as most participants employed the Fraunhofer model, which does not require knowledge of the optical constants for the calculations. This also is reflected that in Table 10 , where data in the lower sizes are often considered outside the $95 \%$ confidence limits.

In LAS-D, since the dispersing medium is air, refractive indices are needed only for the particle phase. All participants who reported a complex refractive index used 0.1 for the imaginary component. This value has also been commonly reported in the literature, but neither the exact origin nor its appropriateness are clearly established. Most of the reported values for the real component were near 1.7: 1.68 ( 3 participants) and 1.70 (4 participants). One participant reported a value of 1.0 , which is clearly too low for a cement powder. Based on the typical composition of portland cements and the known refractive index values for the individual components [8], a value near 1.7 appears appropriate. Again, a procedure for selecting or estimating the refractive indices should be established for any standard method using laser diffraction. 


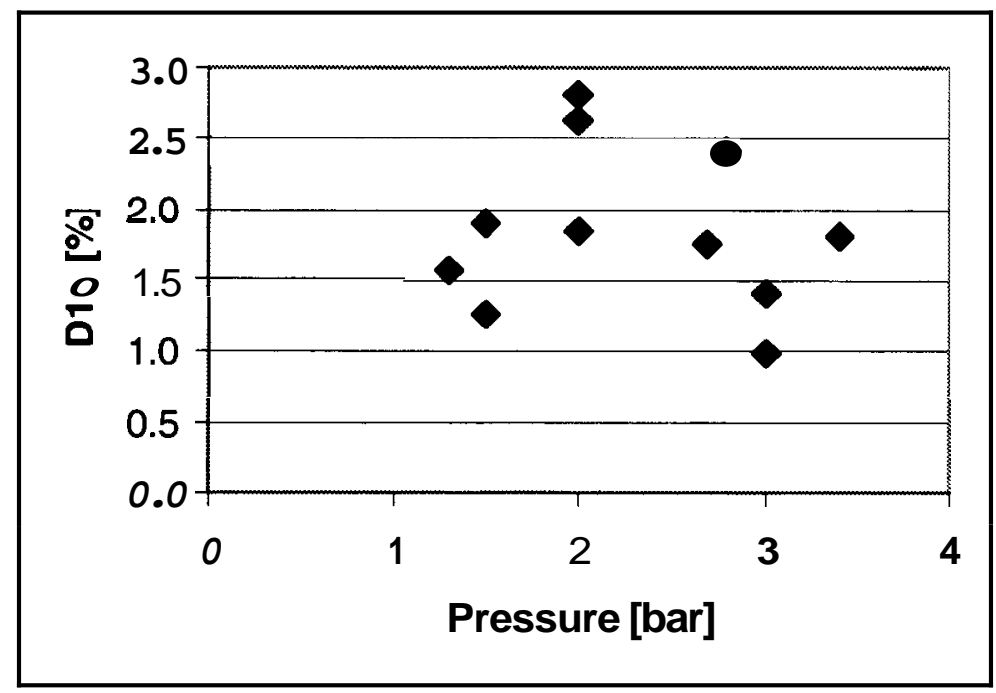

Figure 11: Relationship between the D10 and the pressure used during the measurement in LAS-D.

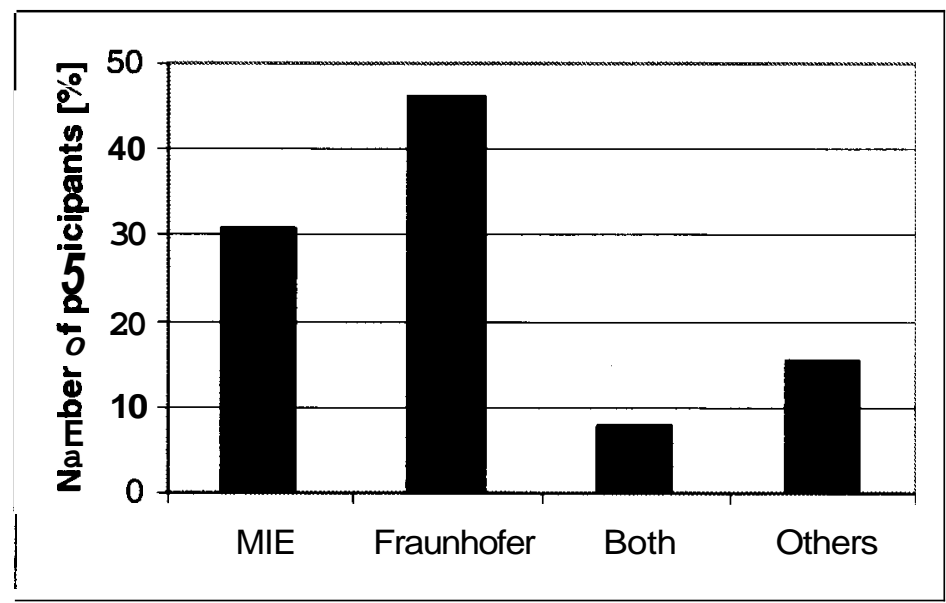

Figure 12: Distribution of the reported optical models used with LAS-D.

\subsubsection{Electrical zone sensing (EZS) and SEM}

The other two measurement methods, EZS and SEM, were used by only three participants in total:

- EZS: 2 participants (\#A15,\#1773)

- SEM: 1 participant (Al)

Therefore, the examination of the method used for dispersing the cement will be based on the details provided by each participant.

Participant \#A15 reported that $1.1 \mathrm{~g}$ of sample was wetted with a few drops of IPA in a small container. Once the sample was completely wet, IPA was added to a total volume of $20 \mathrm{~mL}$ and 
the cuvette was capped. The cuvette was then rocked by hand before being placed in an ultrasonic bath $(\mathbf{1 2 5} \mathrm{W}$ for $15 \mathrm{~s})$. Two drops of the dispersion were added to $100 \mathrm{~mL}$ of electrolyte ( $2 \% \mathrm{NH}_{4} \mathrm{SCN}$ in IPA added as an electrical conductor) with a transfer pipette. The concentration of the dispersion was $0.055 \mathrm{~g} / \mathrm{mL}$. The dispersion was stirred during the measurement using a magnetic stir bar to avoid sedimentation. A single orifice of size $\mathbf{0 . 1 4 0} \mathrm{mm}$ was used during measurements. The measurement was completed in $60 \mathrm{~s}$.

The following information is available from participant \#1773. The concentration of the dispersion was $\mathbf{0 . 0 0 0 1} \mathrm{g} / \mathrm{mL}$ and it was prepared by dilution from a more concentrated stock $(5.0 \mathrm{~g} / \mathrm{mL})$. The medium used was IPA with no additives. The stock dispersion was ultrasonicated, prior to dilution and introduction to the measuring device, for $5 \mathrm{~s}$ at an undetermined output power in a bath sonicator. The orifice used was smaller than $\mathbf{4 0 0} \mu \mathrm{m}$ and the measurement lasted $\mathbf{1 2 0} \mathrm{s}$.

The SEM method was used only by participant \#A1. The general description of the method is given in Section 2.1. The sample preparation is summarized below. The type of medium used for dispersion was ethanol and the duration of the measurement varies depending on knowledge of the sample to be analyzed. It appears that it could take anywhere from $11 \mathrm{~min}$ to about $9 \mathrm{~h}$ to analyze the distribution by SEM. The preparation of the specimen prior to the analysis requires less than $\mathbf{1 0}$ min according to the participant\#AI.

\subsubsection{SEM sample preparation}

This procedure was reported by participant \#A1. The goal in preparing cement samples by the method described here is to obtain a representative portion of an as-received bulk powder sample, redeposit that portion onto a polycarbonate filter, and mount the filter onto a SEM stub for analysis. The procedure is divided in five steps:

1. Preparation of the sample dispersion

2. Redeposition of the solution onto the SEM stub

3. Checking the particle loading on the filter

4. Mounting the filter onto the SEM stub

5. Carbon coating of the sample

The sample dispersion is prepared by taking a representative portion of the as-received bulk sample and mixing it in a beaker with a sufficient amount of acetone. The beaker is sonicated for $1 \mathrm{~min}$ to $3 \mathrm{~min}$ (or longer) to disperse all particles. To deposit the cement onto the SEM stub the following procedure is used. A polycarbonate filter, shiny side up, is placed onto the filtration apparatus, with a funnel placed on top of the filter to secure it. The dispersion prepared above is poured onto the filter apparatus and rinsed with acetone. Immediately the vacuum pump is turned on and the sides of the funnel are rinsed with acetone before the solution has completely filtered. The vacuum pump is left on to partially dry the filter.

To determine that the particles are properly deposited, the filter is examined under a light microscope at $1000 \mathrm{X}$ magnification. The criteria used is that there should be an even loading distribution, i.e., particles are not touching each other, approximately 15 to 20 particles per field. 
Once the filter is dry, a $20 \mathrm{~mm}$ (0.75 in) square piece is cut from the filter, and glued onto the SEM stub using colloidal graphite "dag". After the dag is completely dry, a thin coat of carbon is applied by evaporative deposition.

\subsubsection{Summary}

From the analysis of the methodology reported by the participants, it is clear that most of the participants used a laser diffraction method, either wet or dry. Although there is a wide range of methods used to prepare and analyze the specimen, some tendencies could be found for each method. This could be used for the basis of a draft standard method, at least for one based on laser diffraction.

In LAS-W, the following parameters could be established based on the results of this roundrobin:

- Medium: IPA

- Concentration of the dispersion determined from the obscuration value and not a fixed concentration, or a fixed stock concentration followed by obscuration-based dilution.

On the other hand, a better understanding of the influence of the following parameters on the results should be investigated further:

- Refractive indices (especially the real value)

- The duration and intensity of ultrasonication

Since there were fewer parameters to select for the LAS-D method, the standardization should be less complicated. It is clear that the compressed air method is the most widely used, but this could not be a parameter to be fixed in the ASTM procedure because it depends on the manufacturer and not on the user. The pressure varies somewhat but the median value could be selected or more studies could be made to determine the influence of the pressure on the dispersion of the cement particles. The results are interpreted using the same methods as for LAS-W, i.e., Mie or Fraunhofer or both. As the size of the smallest particles could not be correctly determined using Fraunhofer, the authors suggest that both models be included. As it is not known how the participants selected the refractive index, further research to determine appropriate refractive indices should be conducted.

No clear conclusions could be drawn for the other methods due to the small number of participants using them. 


\section{Recommendations and conclusions}

This report had two goals and therefore there should be two sets of recommendations:

- A reference material

- Standard method to measure PSD of cement

\section{I Reference material}

As SRM $114 \mathrm{p}$ is widely used in the cement industry for calibration of the Blaine measurement, it is an appropriate choice to be used as a reference material for PSD determination. In this report and in the previous one [2], various PSDs were obtained by statistically analyzing the data obtained from round-robins sponsored by ASTM. To obtain a larger data set and to improve the statistical reliability of the results, one could conceivable combine the data from both roundrobins to establish the PSD for SRM 114p. Nevertheless, it should be kept in mind that more than one PSD could be established from the data. The following two PSDs could be made available: 1) LAS-W; 2) LAS-D.

NIST is prepared to combine the data from the two round-robins and to incorporate the results into the certification for SRM 114p as informational data. This could be accomplished in early 2003.

The supplies of SRM 114p available at NIST will cover industry needs for about $\mathbf{1 8}$ months. Therefore, NIST is initiating the procedure for securing and characterizing the next SRM 114. The certificate for the new SRM 114 should also include the PSD of the cement.

\subsection{Standard method}

There are several methods used to measure the PSD of cements. From this report and from the previous report [2], it is clear that the majority of the cement industry uses either LAS-W or LAS-D. Other methods are used by a small percentage of industry only.

In the EZS case, it should be noted that only one participant (out of 2) belonged to the cement industry; the other was an instrument manufacturer. This infers that the cement industry by and large does not use EZS in standard practice. Therefore, a standard method for EZS would not be widely used and would therefore be unsupportable. The third method used in this round-robin, SEM, is in our opinion at the research and development stage, and is also not widely used in industry. Development of a SEM standard method would therefore not be warranted based on current industry use. It would seem that the duration of the measurements and the lengthy preparation procedure would limit SEM use primarily to research or special projects. The designers of this test argue that it could also give the composition of the cement simultaneously with PSD, and this may be the single most important benefit of SEM in this respect. If compositional data were necessary, SEM might prove extremely useful. The authors believe that this information is not necessary for quality control at a cement plant on a daily basis.

Therefore, there is a strong argument for ASTM committee C01.25.01 and NIST to make an investment in time and effort to design a standard test method to measure the PSD of cement 
powder using laser diffraction (both wet and dry). From this report, several parameters could already be narrowed or fixed. A small task group could conceivably help define the next set of specifications to be recommended for a future round-robin as part of the standards development process.

\section{References}

1 "Standard Test Method for Fineness of Portland Cement by the Turbidimeter" ASTM C 115-96, Annual Book of ASTM Standards, Vol. 04.01.

2 Ferraris, C.F, Hackley V.A., Aviles A.I., Buchanan C.E., "Analysis of the ASTM RoundRobin Test on Particle Size Distribution of Portland Cement: Phase I" NISTIR 6883, May 2002.

3 Jillavenkatesa A., Dapkunas S. J., Lum L.-S. H., "Particle Size Characterization,” NIST Special Publication 960-1,2001.

4 A.J. Schwoeble, A.M. Dalley, B.C. Henderson, G.S. Casuccio, "Computer-Controlled SEM and Microimaging of Fine Particles,” J. Metals, pp. I 1-14, 1988.

5 G.S. Casuccio, P.B. Janocko, R.J. Lee, J.F. Kelly, S.L. Dattner, J.S. Mgebroff, “The Use of Computer Controlled Scanning Electron Microscopy in Environmental Studies," APCA Journal, 33 [I0], pp. 937-943, 1983.

6 S.K. Kennedy, W. Walker, and B. Forslund, "Speciation and Characterization of Heavy Metal-Contaminated Soils Using Computer-Controlled Scanning Electron Microscopy," in Press 2002.

7 ISO 13320-1:1999(E), "Particle size analysis - Laser diffraction methods - Part 1: General principles".

8 Cyr, M. and Tagnit-Hamou, A., "Particle size distribution of fine powders by Laser diffraction spectrometry. Case of cementitious materials," Muter Struct, 34, pp. 342-350, 2001. 
APPENDICES 
A-32 


\section{TABLE OF CONTENT}

APPENDIX A: CEMENT CHARACTERISTICS

APPENDIX B: PSD DATA RECEIVED FOR THE CEMENTS CCRL 143 AND 144.....42

APPENDIX C: FORM RETURNED BY PARTICIPANTS _ _ $\quad 49$

APPENDIX D: SPECIFICATION FOR THE TO TESTS SRM114P_— $\quad \mathbf{5 6}$

APPENDIX E-1: PARTICLE SIZE DISTRIBUTION USING ONLY DATA FROM LASER WET

APPENDIX E-2: PARTICLE SIZE DISTRIBUTION USING ONLY DATA FROM LASER DRY 
A-34 


\title{
Appendix A: Cement characteristics'
}

\author{
CCRL PROFICIENCY SAMPLE PROGRAM
}

Portland Cement Proficiency Samples No. 143 and No. 144

Final Report - Heat of Hydration Results

March 22,2002

SUMMARY OF RESULTS

Sample No. 143

Sample No. 144

\begin{tabular}{|c|c|c|c|c|c|c|c|c|}
\hline Test & & \#Labs & Average & S.D. & C.V. & Average & S.D. & C.V. \\
\hline Heat Solution Dry & $\mathrm{caVg}$ & 19 & 604.3 & 20.3 & 3.35 & 597.2 & 10.4 & 1.74 \\
\hline Heat Solution Dry & $\mathrm{cal} / \mathrm{g}$ & 18 & 599.7 & 3.9 & 0.645 & 599.3 & 5.3 & 0.882 \\
\hline Heat Sol 7-day & $\mathrm{caVg}$ & 19 & 528.8 & 24.3 & 4.59 & 521.8 & 7.4 & 1.41 \\
\hline Heat Sol 7-day & $\mathrm{caVg}$ & 18 & 523.3 & 5.5 & 1.06 & 523.0 & 5.4 & 1.03 \\
\hline Heat Sol 28-day & $\mathrm{cal} / \mathrm{g}$ & 11 & 502.9 & 40.6 & 8.08 & 499.3 & 45.6 & 9.14 \\
\hline Heat Sol 28-day & $\mathrm{caVg}$ & 10 & 515.0 & 6.0 & 1.165 & 513.0 & 4.2 & 0.825 \\
\hline Heat Hyd 7-day & $\mathrm{caVg}$ & 19 & 75.7 & 5.9 & 7.76 & 75.6 & 6.9 & 9.12 \\
\hline Heat Hyd 28-day & $\mathrm{caVg}$ & 11 & 86.2 & 6.5 & 7.55 & 86.9 & 5.6 & 6.40 \\
\hline
\end{tabular}

* ELIMINATEDLABS: Data over three S.D. from the mean

$\begin{array}{ll}\text { Heat Solution Dry } & 1916 \\ \text { Heat Solution 7-day } & 1916 \\ \text { Heat Solution 28-day } & 557\end{array}$

\footnotetext{
${ }^{\text {I }}$ The policy of the National Institute of Standards and Technology is to use the International System of Units (metric units) on all its publications. In this appendix however, all the tables are reproduced, with permission, and as published by CCRL which describes measurements in certain non-SI units.
} 
CCRL PROFICIENCY SAMPLE PROGRAM

Portland Cement Proficiency Samples No. 143 and No. 144

Final Report - Chemical Rapid Method Results

March 22,2002

\section{SUMMARY OF RESULTS}

Sample No. 143

Sample No. 144

\begin{tabular}{|c|c|c|c|c|c|c|c|c|}
\hline Test & & \#Labs & Average & S.D. & C.V. & Average & S.D. & C.V. \\
\hline Silicon Dioxide & prent & 176 & 21.216 & 0.25 & 1.16 & 20.366 & 0.24 & 1.17 \\
\hline Silicon Dioxide & prent & * 172 & 21.213 & 0.22 & 1.06 & 20.359 & 0.20 & 1.00 \\
\hline Aluminum Oxide & prent & 184 & 4.472 & 0.18 & 3.96 & 5.306 & 0.18 & 3.35 \\
\hline Aluminum Oxide & prent & * 179 & 4.465 & 0.15 & 3.35 & 5.311 & 0.12 & 2.33 \\
\hline Ferric Oxide & prent & 183 & 3.521 & 0.071 & 2.02 & 2.354 & 0.130 & 5.52 \\
\hline Ferric Oxide & prent & * 178 & 3.527 & 0.057 & 1.62 & 2.346 & 0.060 & 2.56 \\
\hline Calcium Oxide & prent & 177 & 63.781 & 0.65 & 1.02 & 64.551 & 0.66 & 1.02 \\
\hline Calcium Oxide & prent & * 172 & 63.765 & 0.39 & 0.616 & 64.607 & 0.45 & 0.693 \\
\hline Magnesium Oxide & prent & 185 & 1.689 & 0.18 & 10.4 & 0.980 & 0.18 & 18.6 \\
\hline Magnesium Oxide & prent & * 172 & 1.678 & 0.055 & 3.28 & 0.960 & 0.060 & 6.20 \\
\hline Sulfur Trioxide & prent & 172 & 2.728 & 0.13 & 4.65 & 2.998 & 0.26 & 8.66 \\
\hline Sulfur Trioxide & prent & $* 165$ & 2.728 & 0.084 & 3.07 & 3.020 & 0.104 & 3.43 \\
\hline
\end{tabular}

* ELIMINATEDLABS: Data over three S.D. from the mean

Silicon Dioxide

Aluminum Oxide

Ferric Oxide

Calcium Oxide

Magnesium Oxide

Sulfur Trioxide
93501547690

132142501504547

142360493501787

$2356360547 \quad 1715$

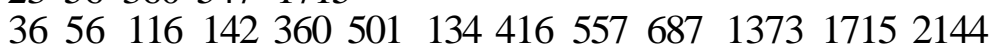

$56 \quad 6871211426929181053$ 


\section{CCRL PROFICIENCY SAMPLE PROGRAM}

Portland Cement Proficiency Samples No. 143 and No. 144

Final Report - Chemical Rapid Method Results

March 22,2002

\section{SUMMARY OF RESULTS}

Sample No. 143

Sample No. 144

\begin{tabular}{|c|c|c|c|c|c|c|c|c|}
\hline Test & & \#Labs & Average & S.D. & C.V. & Average & S.D. & C.V. \\
\hline Loss on Ignition & prent & * 131 & 1.525 & 0.16 & 10.21 & 2.153 & 0.14 & 6.39 \\
\hline Loss on Ignition & prent & * 126 & 1.522 & 0.103 & 6.79 & 2.151 & 0.093 & 4.33 \\
\hline Sodium Oxide & prent & 176 & 0.132 & 0.11 & 82.0 & 0.131 & 0.16 & 125.4 \\
\hline Sodium Oxide & prent & * 171 & 0.122 & 0.024 & 19.8 & 0.114 & 0.027 & 23.9 \\
\hline Potassium Oxide & prent & $\begin{array}{r}184 \\
* \quad 1\end{array}$ & 0.711 & 0.068 & 9.61 & 0.750 & 0.058 & 7.72 \\
\hline Potassium Oxide & prent & * 175 & 0.722 & 0.021 & 2.95 & 0.760 & 0.026 & 3.37 \\
\hline Manganic Oxide & prent & 81 & 0.061 & 0.19 & 309 & 0.060 & 0.11 & 184 \\
\hline Manganic Oxide & prent & 80 & 0.040 & 0.013 & 32.1 & 0.048 & 0.014 & 28.5 \\
\hline Phosphorus Pent & prent & * 113 & 0.055 & 0.027 & 48.9 & 0.101 & 0.026 & 25.4 \\
\hline Phosphorus Pent & prent & * 107 & 0.050 & 0.0110 & 21.8 & 0.095 & 0.0096 & 10.0 \\
\hline Titanium Dioxide & prent & 127 & 0.215 & 0.040 & 18.5 & 0.309 & 0.044 & 14.5 \\
\hline Titanium Dioxide & prent & * 122 & 0.217 & 0.018 & 8.27 & 0.310 & 0.027 & 8.70 \\
\hline
\end{tabular}

${ }^{*}$ ELIMINATEDLABS: Data over three S.D. from the mean

Loss on Ignition

Sodium Oxide

Potassium Oxide

Manganic Oxide

Phosphorus Pentoxide

Titanium Dioxide
$56 \quad 181438450996$

$\begin{array}{lllll}56 & 36 & 547 & 698 & 1373\end{array}$

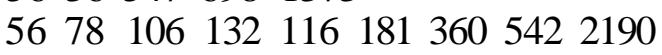

619

1271421814925021196

124166181438502 
CCRL PROFICIENCY SAMPLE PROGRAM

Portland Cement Proficiency Samples No. 143 and No. 144

Final Report - Physical Results

March 22,2002

SUMMARY OF RESULTS

Sample No. 143

Sample No. 144

\begin{tabular}{|c|c|c|c|c|c|c|c|c|}
\hline Test & & \#Labs & Average & S.D. & C.V. & Average & S.D. & C.V. \\
\hline N.C. Water & prent & 236 & 25.08 & 1.5 & 6.12 & 24.66 & 1.6 & 6.29 \\
\hline N.C. Water & prent & ${ }^{*} 234$ & 25.18 & 0.42 & 1.67 & 24.77 & 0.42 & 1.68 \\
\hline Vicat TS Initial & $\min$ & 224 & 121 & 15.3 & 12.6 & 92 & 18.2 & 19.8 \\
\hline Vicat TS Initial & $\min$ & ${ }^{*} 218$ & 120 & 13.6 & 11.3 & 90 & 12.9 & 14.3 \\
\hline Vicat TS Final & $\min$ & 220 & 216 & 33.7 & 15.6 & 173 & 32.2 & 18.6 \\
\hline Vicat TS Final & $\min$ & $* 216$ & 217 & 30.7 & 14.2 & 173 & 31.5 & 18.3 \\
\hline Gillmore TS Initial & $1 \mathrm{~min}$ & 176 & 151 & 22.7 & 15.0 & 122 & 23.8 & 19.4 \\
\hline Gillmore TS Initial & $1 \mathrm{~min}$ & * 173 & 151 & 21.8 & 14.5 & 121 & 18.7 & 15.5 \\
\hline Gillmore TS Final & $\min$ & * 174 & 245 & 36.7 & 15.0 & 200 & 34.6 & 17.3 \\
\hline Gillmore TS Final & $\min$ & * 172 & 245 & 34.1 & 13.9 & 200 & 33.8 & 16.9 \\
\hline False Set & prent & 193 & 82.1 & 8.1 & 9.85 & 73.0 & 11.3 & 15.51 \\
\hline Autoclave Expan & prent & 219 & -0.009 & 0.040 & -446.22 & 0.031 & 0.063 & 202.90 \\
\hline Autoclave Expan & prent & $* 216$ & -0.007 & 0.022 & -312.68 & 0.032 & 0.027 & 84.48 \\
\hline Air Content & prent & 219 & 8.46 & 1.1 & 13.3 & 10.79 & 1.3 & 12.1 \\
\hline Air Content & prent & ${ }^{*} 217$ & 8.49 & 1.1 & 12.5 & 10.83 & 1.2 & 11.4 \\
\hline AC Mix Water & prent & 218 & 67.87 & 7.0 & 10.4 & 66.79 & 7.1 & 10.6 \\
\hline AC Mix Water & prent & ${ }^{*} 212$ & 68.29 & 2.3 & 3.39 & 67.20 & 2.8 & 4.18 \\
\hline AC Flow & prent & 217 & 88.0 & 3.6 & 4.04 & 89.3 & 3.3 & 3.70 \\
\hline AC Flow & prent & ${ }^{*} 216$ & 88.1 & 3.5 & 3.97 & 89.4 & 3.3 & 3.71 \\
\hline
\end{tabular}

CONTINUED ON REVERSE SIDE

* ELIMINATED LABS: Data over three S.D. from the mean

\begin{tabular}{|c|c|}
\hline Normal Consistency & 201221 \\
\hline Vicat TS Initial & $\begin{array}{llllll}36 & 49 & 819 & 1190 & 1483 & 1644\end{array}$ \\
\hline Vicat TS Final & $11 \quad 1241561190$ \\
\hline Gillmore TS Initial & 124252996 \\
\hline Gillmore TS Final & 124270 \\
\hline Autoclave Expansion & 1515261819 \\
\hline Air Content & 3602144 \\
\hline Air Content Mix Water & $\begin{array}{llllll}354 & 127 & 360 & 1523 & 1956 & 2144\end{array}$ \\
\hline Air Content Flow & 886 \\
\hline
\end{tabular}


CCRL PROFICIENCY SAMPLE PROGRAM

Portland Cement Proficiency Samples No. 143 and No. 144

Final Report - Physical Results

March 22,2002

SUMMARY OF RESULTS

Sample No. 143

Sample No. 144

\begin{tabular}{|c|c|c|c|c|c|c|c|c|}
\hline Test & & \#Labs & Average & S.D. & C.V. & Average & S.D. & C.V. \\
\hline Comp Str 3-day & psi & 242 & 3862 & 284.2 & 7.36 & 3608 & 283.7 & 7.86 \\
\hline Comp Str 3-day & psi & * 238 & 3871 & 266.8 & 6.89 & 3611 & 234.8 & 6.50 \\
\hline Comp Str 7-day & psi & 241 & 4693 & 301.6 & 6.42 & 4456 & 294.7 & 6.61 \\
\hline Comp Str 7-day & psi & * 239 & 4689 & 296.6 & 6.32 & 4445 & 271.9 & 6.12 \\
\hline Comp Str 28-day & psi & 201 & 5933 & 391.6 & 6.60 & 5510 & 360.3 & 6.54 \\
\hline Comp Str 28-day & psi & * 197 & 5932 & 375.8 & 6.33 & 5499 & 323.6 & 5.88 \\
\hline CS Flow & prent & 201 & 121.4 & 11.5 & 9.49 & 115.0 & 10.7 & 9.28 \\
\hline Fineness AP & $\mathrm{cm}^{2} / \mathrm{g}$ & 239 & 3979 & 150.1 & 3.77 & 4122 & 123.7 & 3.00 \\
\hline Fineness AP & $\mathrm{cm}^{2} / \mathrm{g}$ & * 229 & 3982 & 79.9 & 2.01 & 4123 & 83.5 & 2.02 \\
\hline Fineness WT & $\mathrm{cm}^{2} / \mathrm{g}$ & 33 & 2237 & 113.5 & 5.08 & 2128 & 110.7 & 5.20 \\
\hline Fineness WT & $\mathrm{cm}^{2} / \mathrm{g}$ & * 32 & 2249 & 92.6 & 4.12 & 2136 & 101.3 & 4.74 \\
\hline 45ym sieve & prent & 216 & 96.586 & 0.81 & 0.840 & 91.161 & 1.20 & 1.315 \\
\hline 45ym sieve & prent & * 211 & 96.683 & 0.46 & 0.472 & 91.153 & 0.93 & 1.024 \\
\hline
\end{tabular}

* ELIMINATED LABS: Data over three S.D. from the mean

Comp Strength 3-day $\quad 14301521053$

Comp Strength 7-day 301657

Comp Strrengh 28-day 303812511657

Fineness Air Perm $\quad 222346492834311025105319162144$

Fineness Wagner Turb 787

$45 \mu \mathrm{m}$ Sieve $\quad 802072658862144$ 
CCRL Proficiency Sample Program

Portland Cement Proficiency Samples No. 143 and No. 144

Final Report - Chemical Results

March 22,2002

SUMMARY OFRESULTS

Sample No. 143

Sample No. 144

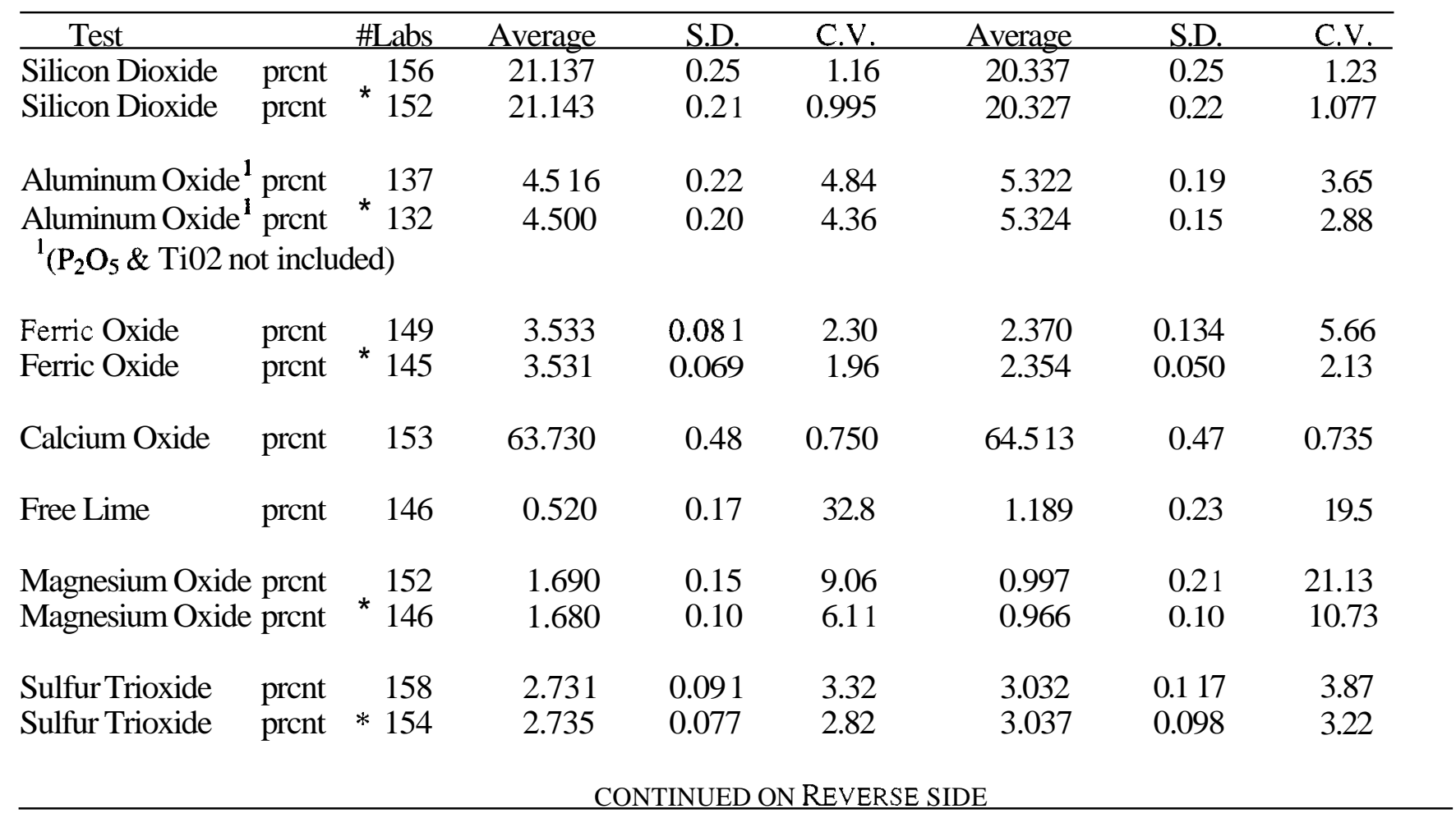

* ELIMINATED LABS: Data over three S.D. from the mean

Silicon Dioxide

Aluminum Oxide Ferric Oxide Magnesium Oxide Sulfur Trioxide
1161784921526

$98 \quad 142413 \quad 501 \quad 1526$

$142501 \quad 10251526$

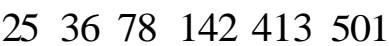

116121142918 
CCFU Proficiency Sample Program

Portland Cement Proficiency Samples No. 143 and No. 144

Final Report - Chemical Results

March 22,2002

SUMMARY OF RESULTS

Sample No. 143

Sample No. 144

\begin{tabular}{|c|c|c|c|c|c|c|c|c|}
\hline Test & & \#Labs & Average & S.D. & C.V. & Average & S.D. & C.V. \\
\hline Loss on Ignition & prent & 186 & 1.508 & 0.11 & 7.15 & 2.142 & 0.11 & 5.00 \\
\hline Loss on Ignition & prent & * 179 & 1.511 & 0.090 & 5.94 & 2.152 & 0.086 & 3.99 \\
\hline Insoluble Residue & prent & 174 & 0.323 & 0.220 & 68.3 & 0.392 & 0.096 & 24.5 \\
\hline Insoluble Residue & prent & * 168 & 0.294 & 0.076 & 25.9 & 0.389 & 0.088 & 22.8 \\
\hline Sodium Oxide & prent & 129 & 0.123 & 0.038 & 30.5 & 0.112 & 0.034 & 30.4 \\
\hline Sodium Oxide & prent & * 124 & 0.121 & 0.024 & 19.7 & 0.111 & 0.026 & 23.4 \\
\hline Potassium Oxide & prent & 133 & 0.718 & 0.032 & 4.41 & 0.755 & 0.044 & 5.90 \\
\hline Potassium Oxide & prent & * 128 & 0.719 & 0.028 & 3.86 & 0.757 & 0.032 & 4.24 \\
\hline Phosphorus Pent & prent & 69 & 0.060 & 0.061 & 102.5 & 0.110 & 0.107 & 97.1 \\
\hline Phosphorus Pent & prent & * 67 & 0.050 & 0.014 & 28.6 & 0.097 & 0.019 & 19.5 \\
\hline Titanium Dioxide & prent & 79 & 0.217 & 0.031 & 14.5 & 0.345 & 0.326 & 94.6 \\
\hline Titanium Dioxide & prent & * 76 & 0.218 & 0.015 & 7.04 & 0.312 & 0.021 & 6.61 \\
\hline
\end{tabular}

Loss on Ignition

Insoluble Residue

Sodium Oxide

Potassium Oxide

Phosphorus Pentoxide

Titanium Dioxide
* ELIMINATED LABS: Data over three S.D. from the mean

20622145069499619362191

3660931546941379

13441397512511373

35421251782190

96502

96166502 


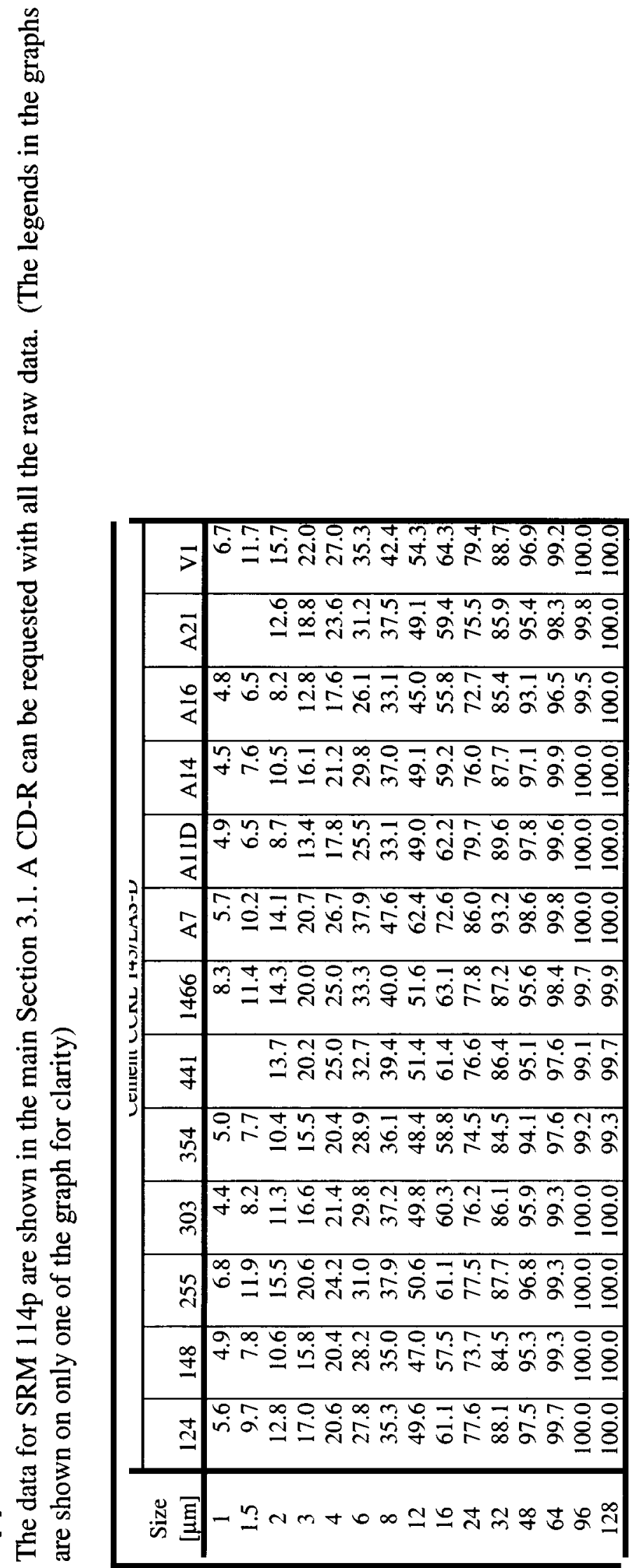




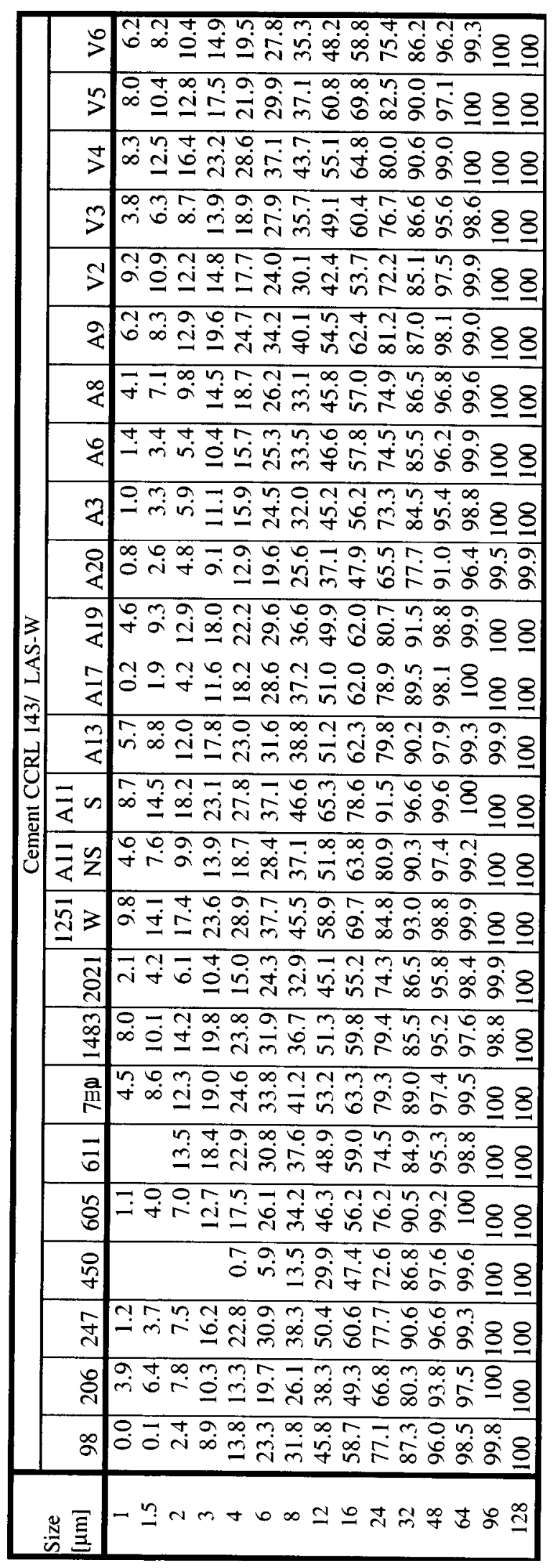




\begin{tabular}{|c|c|c|}
\hline \multicolumn{3}{|r|}{ 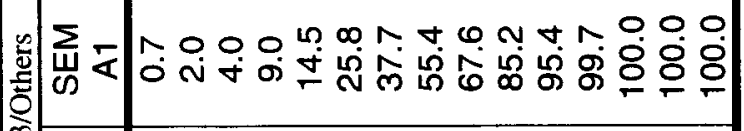 } \\
\hline $\begin{array}{l} \pm \\
\vec{\alpha} \\
\mathcal{\alpha} \\
\mathcal{U}\end{array}$ & W & 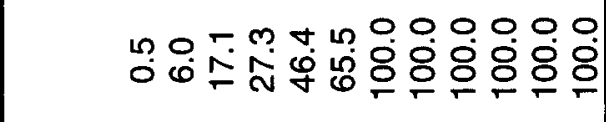 \\
\hline 节 & $\stackrel{n}{N}$ & 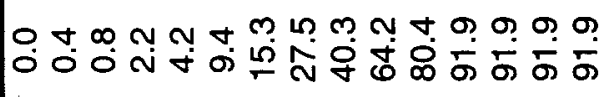 \\
\hline & $\stackrel{\mathbb{N}}{\Xi}$ & 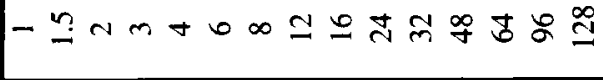 \\
\hline
\end{tabular}




\begin{tabular}{|c|c|}
\hline & 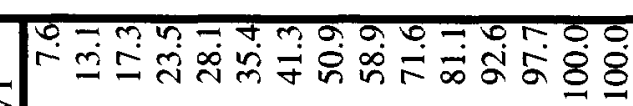 \\
\hline- & 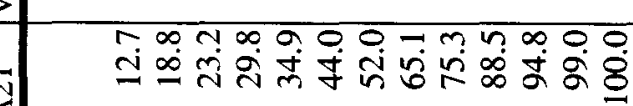 \\
\hline & a \\
\hline & 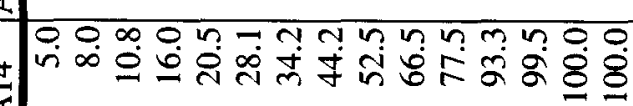 \\
\hline & 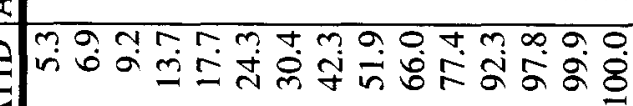 \\
\hline & $\mid$ \\
\hline & 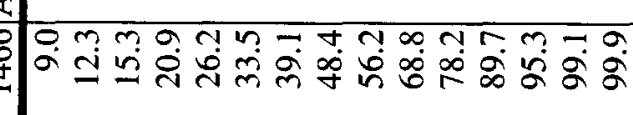 \\
\hline & 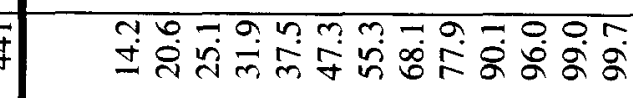 \\
\hline & 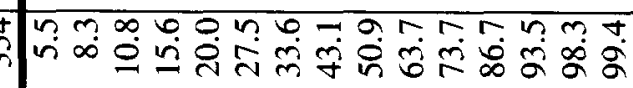 \\
\hline$\overline{\check{c}}$ & 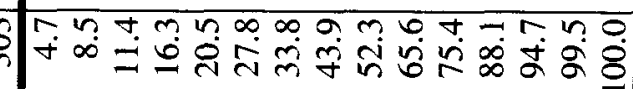 \\
\hline & 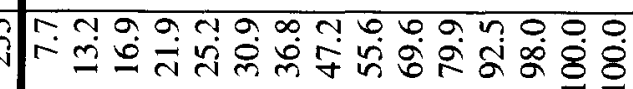 \\
\hline $\bar{d}$ & 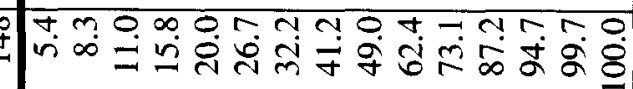 \\
\hline$\check{g}$ & 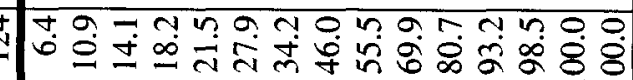 \\
\hline 兽 & 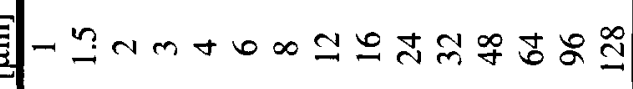 \\
\hline
\end{tabular}




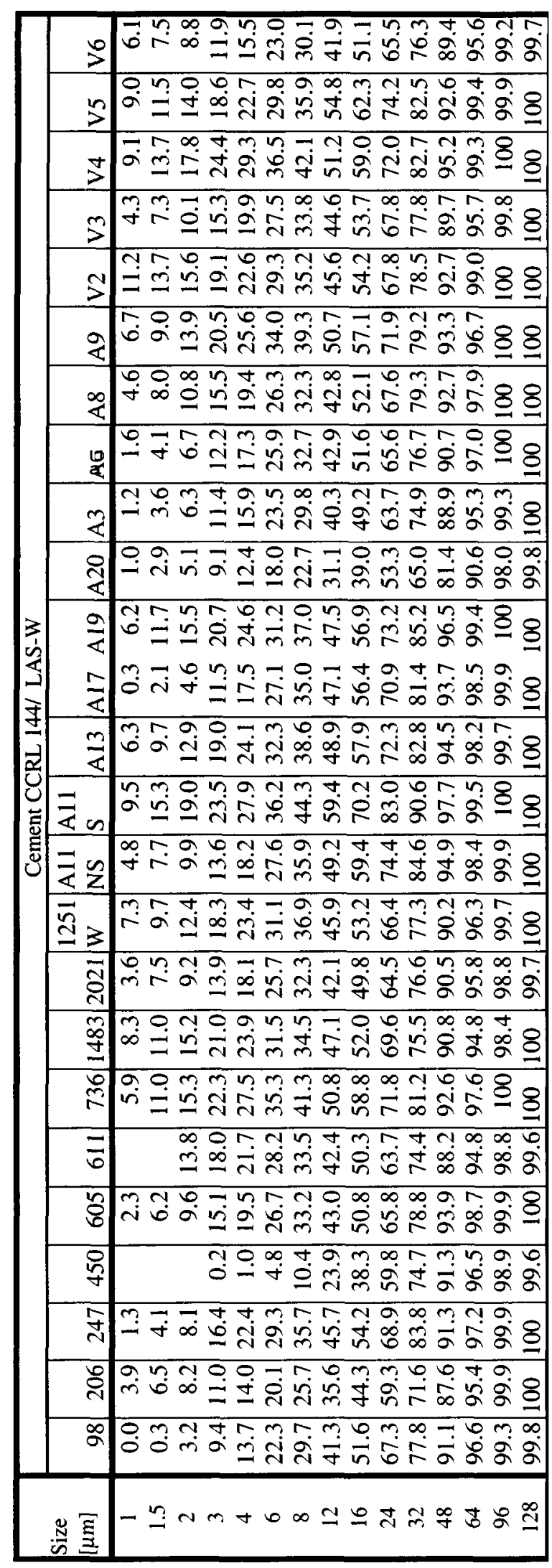




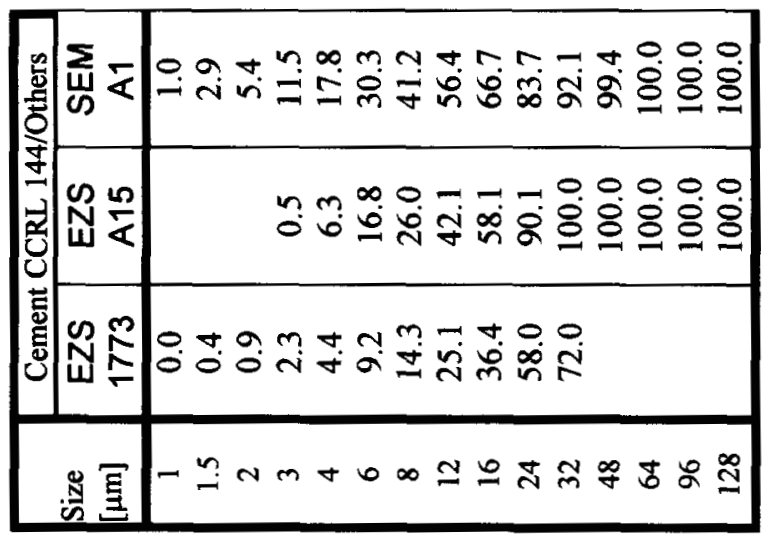




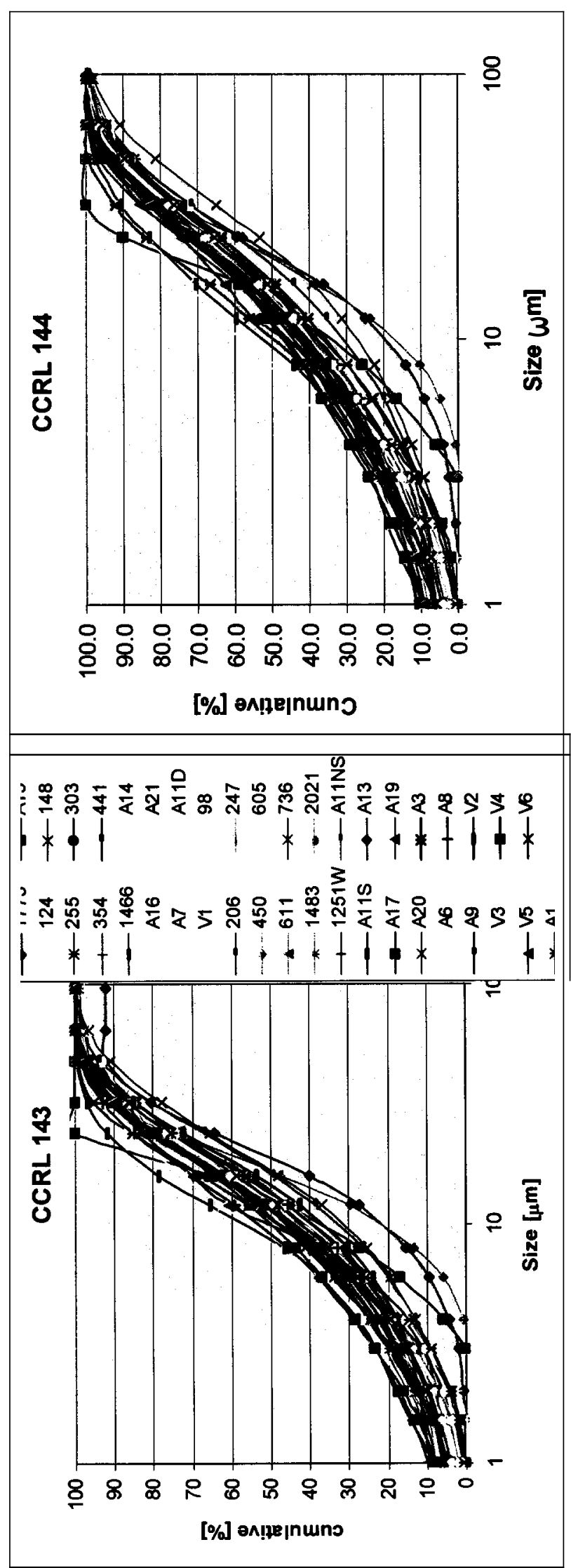




\section{Appendix C: Form returned by participants}

Form to be returned with your results

\begin{tabular}{|l|}
\hline Identification: (the information in this section will be kept confidential) \\
Laboratory name: \\
\hline Address: \\
\hline Contact person: \\
Operator: $\quad$ Phone: \\
Date of the tests: \\
\hline
\end{tabular}

METHOD USED (select one and then go to the section indicated)

口 Laser Diffraction (wet): specimen dispersed in a liquid

口 Laser Diffraction (dry): specimen dispersed in air

Section A

- SEM

口 Particle Counting Technique (EZS, SPOS)

Section B

- Sieve and Sedimentation

Section C

Section D

Section E

\section{Please use the appropriate section(s) corresponding to your method.}

Return all relevant sections by March 10,2002 to:

Clarissa Ferraris (e-mail: clarissa@nist.gov; Fax: 301-9906891)

\section{AND}

Charles Buchanan (e-mail: charlesjr@ roanind.com; Fax: 828-688 5855)

Note: The MS EXCEL spreadsheet containing all the PSD data should be e-mailed back, the forms can be faxed or e-mailed.

\section{Assigned code:}




\section{SECTION A: Laser Diffraction (wet): specimen dispersed in a liquid}

Device brand and model:

Reference material used (if any):

\section{Sample preparation:}

Medium used (circle one): $\quad$ Methanol Ethanol Isopropanol (IPA)

other (specify):

$>$ Concentration of the dispersion: $[\mathrm{g} / \mathrm{mL}]$ Diluted from more concentrated stock? yes no If yes, give stock concentration $[\mathrm{g} / \mathrm{L}]$ :

- Note: use particle density of $\mathbf{3 . 2} \mathrm{g} / \mathrm{mL}$ for calculation of solids concentration. Also indicate density used for medium $[\mathrm{g} / \mathrm{mL}]$ :

Was a surfactant used? (circle one) Yes No

if yes, please specify name and dosage:

Ultrasonication of sample suspension (circle one): $\quad$ Yes No

If yes, please specify intensity and duration:

Was ultrasonic treatment performed (circle one):

(a) inside PSD device; (b) prior to introduction into device; (c) both

- If (b) or (c), please identify type of external ultrasonicator used (circle one) bath submersiblehorn

- If (b) or (c), was the external ultrasonication performed on a (circle one) concentrate or dilute dispersion*

*refers to a suspension at or near the solids concentration used in the actual measurement

\section{Test and results:}

$>$ Duration of the measurement in the PSD device [sec]:

Complex refractive index used for powder: Real: Imaginary:

Refractive index (real) used for medium:

$>$ Model used to interpret the results: (circle one): Mie Fraunhofer Both

Notes: (add any information that could be useful to better describe the procedure used): 
SECTION B: Laser Diffraction (Dry): specimen dispersed in air

Device brand and model:

Reference material used (if anv):

Particle dispersion:

Dispersion procedure: (circle one)

compressed air vacuum

If compressed air, pressure setting used [bar]

Test and results:

$>$ Duration of the measurement in the PSD device [s]

$>$ Give the Refractive index used: Real:

Imaginary:

Model used to interpret the results: (circle one): Mie Fraunhofer

Notes: (add any information that could be useful to better describe the procedure used): 


\section{SECTION C: Scanning Electron Microscopy (SEM)}

Device brand and model:

$\underline{\text { Reference material used (if any): }}$

\section{Sample preparation:}

Concentration of the dispersion: $[\mathrm{g} / \mathrm{mL}]$

$>$ Medium used (circle one): Methanol Ethanol Isopropanol (IPA)

Other (specify):

- Note: use particle density of $3.2 \mathrm{~g} / \mathrm{mL}$ for calculation of solids concentration. Also indicate density used for medium $[\mathrm{g} / \mathrm{mL}]$ :

Was a surfactant used? (circle one) Yes No

if yes, please specify name and dosage:

Ultrasonication of sample suspension (circle one): $\quad$ Yes No

- If yes, please specify intensity and duration:

- If yes, please identify type of ultrasonicator used (circle one)

bath submersible horn

\section{Test and results:}

Number of fields analyzed:

$>$ Number of particles measured per field:

$>$ Magnification used and range of related PSD:

Duration of the measurement in the PSD device [s]:

Describe the method used to interpret the results:

Notes: (add any information that could be useful to better describe the procedure used): 


\section{SECTION D: Particle Counting [Electrical Zone Sensing (EZS) or Single Particle Optical Sensing (SPOS)]}

Device brand and model:

Reference material used (if anv):

\section{Sample ureuaration:}

Concentration of the dispersion $[\mathrm{g} / \mathrm{mL}]$ : Diluted from more concentrated

stock? yes no If yes, give stock concentration $[\mathrm{g} / \mathrm{L}]$ :

Medium used (circle one): Methanol Ethanol Isopropanol (IPA)

Other (specify):

- Note: use particle density of $3.2 \mathrm{~g} / \mathrm{mL}$ for calculation of solids concentration. Also indicate density used for medium $[\mathrm{g} / \mathrm{mL}]$ :

Was a surfactant used? (circle one) Yes No

if yes, please specify name and dosage:

Ultrasonication of sample suspension (circle one): $\quad$ Yes $\quad$ No

If yes, please specify intensity and duration:

Was ultrasonic treatment performed (circle one):

(a) inside PSD device; (b) prior to introduction into device; (c) both

- If (b) or (c), please identify type of external ultrasonicator used (circle one) bath submersible horn

- If (b) or (c), was the external ultrasonication performed on a (circle one) concentrate or dilute dispersion"

"refers to a suspension at or near the concentration used in the actual measurement

\section{Test and results:}

Size of the orifice(s) $[\mathrm{mm}]$ :

Duration of the measurement in the PSD device [s]:

Describe the method used to interpret the results:

Notes: (add any information that could be useful to better describe the procedure used): 


\section{SECTION E: Sieving and Sedimentation}

Was sieving done before sedimentation? Yes No

If yes, please, also answer questions in the section Sieving, if not proceed directly to the section Sedimentation

\section{Sieving}

Device brand and model:

Reference material used (if any):

How was the cement measured (circle one): dry wet

If wet, proceed to Part I; ifdrv, proceed to Part II

PART I: Sieving of liquid-dispersed powders (wet sieving)

Sample preparation

$>$ Medium used (Circle one) Methanol Ethanol Isopropanol Other

(specify):

$>$ Concentration of the dispersion: $[\mathrm{g} / \mathrm{mL}]$

- Note: use particle density of $\mathbf{3 . 2} \mathrm{g} / \mathrm{mL}$ for calculation of solids concentration. Also indicate density used for medium $[\mathrm{g} / \mathrm{mL}]$ :

Was a surfactant used? Yes No,

if yes please specify name and dosage

Ultrasonication of sample suspension (circle one): $\quad$ Yes No

If yes, please specify intensity and duration:

Was ultrasonic treatment performed (circle one):

(a) during sieving; (b) prior to sieving; (c) both

- If (b) or (c), please identify type of external ultrasonicator used (circle one)

bath submersible horn

Test and results:

$>$ Type of sieving procedure used (circle one): manual automated

$>$ Number of sieves used:

Size of the sieves used (use the ASTM E1 1 designations):

Describe the method used to interpret the results:

PART II: Sieving of drypowders (dry sieving)

$>$ If applicable, describe how powder was dispersed prior to sieving: 
Type of sieving method used (circle one): manual automated

Duration of the test [sec]:

Number of sieves used:

Size of the sieves used (use the ASTM E1 1 designations):

Describe the method used to interpret the results:

\section{Sedimentation}

Was the Sedimentation method (circle one): gravitational or centrifugal ?

Device brand and model:

Reference material used (if any):

\section{Sample preparation:}

$>$ Medium used (Circle one) Methanol Ethanol Isopropanol Other

(specify):

Concentration of the dispersion: $[\mathrm{g} / \mathrm{mL}]$

- Note: use particle density of $3.2 \mathrm{~g} / \mathrm{mL}$ for calculation of solids concentration. Also

indicate density used for medium $[\mathrm{g} / \mathrm{mL}]$ :

Was a surfactant used? Yes No,

if yes please specify name and dosage

Ultrasonication of sample suspension (circle one): Yes No

If yes, please specify intensity and duration:

If yes, please identify type of ultrasonicator used (circle one)

bath

submersible horn

\section{Test and results:}

$>$ Particle density used $(\mathrm{g} / \mathrm{mL})$ : measured? yes no

if yes, by what method?

Detection method used: (circle one)

$$
\text { optical } \quad \mathrm{X} \text {-ray cumulative mass }
$$

Duration of the measurement [sec]:

Notes: (add any information that could be useful to better describe the procedure used): (use the back of the page if needed) 


\section{APPENDIX D: Specification for the to tests SRM114p.}

\section{SECTION A: Laser Diffraction (wet) - specimen dispersed in a liquid}

\section{Sample preparation procedure:}

Dispersion medium: Isopropanol (IPA) (density: $0.7855 \mathrm{~g} / \mathrm{mL}$ at 20 "C)

$>\underline{\text { No }}$ surfactants should be used for dispersion of the cement powder.

$>$ Use a standard Pyrex glass beaker to prepare stock suspension

Prepare stock concentrate at solids concentration $[\mathrm{g} / \mathrm{L}]: 85$

- Add $10 \mathrm{~g}$ cement powder to $90 \mathrm{~g}(115 \mathrm{~mL})$ of IPA

Ultrasonicate stock concentrate prior to measurement:

- If available, use an external, submersible-horn-type ultrasonic disruptor

- Ultrasonicate on a medium setting for 1 min duration

- Indicate make \& model of ultrasonicator device:

- Indicate output power setting used [watts]:

- If submersible-typeis not available, use an ultrasonic bath

- Place beaker with sample in bath and use the highest setting available for $5 \mathrm{~min}$ duration:

- Indicate make \& model of ultrasonicator device:

\section{Testand results:}

Run measurements at or near 20 "C. If measurement temperature deviates by more than \pm 2 "C from 20 "C, then indicate temperature here:
Refractive index values for powder: Real:
1.70 Imaginary: 0.1

Refractive index value for medium: 1.378 (indicate if instrument requires that you use a different value for RI):

For measurements, use drop-wise additions of concentrate to particle-free IPA circulating within the measurement device until the appropriate obscuration level (i.e., particle concentration) is obtained according to the instrument manufacturer's recommendations.

$>$ Indicate duration of the measurement in the PSD device [s]:

Use model to interpret the results (circle one): Mie Fraunhofer Both

Note: if available, analyze data using both models separately, and provide separate sets of results for each.

Notes: (add any information that could be useful to better describe the procedure used, use additional blank sheets if necessary): 


\section{SECTION B: Laser Diffraction (Dry): specimen dispersed in air}

Particle dispersion:

Dispersion procedure: (circle one) compressed air vacuum

If compressed air, use manufacturer's recommended pressure setting, and indicate here [bar] .

Test and results:

$>$ Refractive index values for powder: Real: $\quad 1.70 \quad$ Imaginary: 0.1

$>$ Use manufacturer's recommendations for amount of powder to introduce into device, and use recommended values for parameters not specifically indicated here.

Duration of the measurement in the PSD device [s]:

$>$ Use model to interpret the results (circle one): Mie Fraunhofer Both

Note: $f$ available, analyze data using both models separately, and provide separate sets of results for each.

Notes: (add any information that could be useful to better describe the procedure used): 


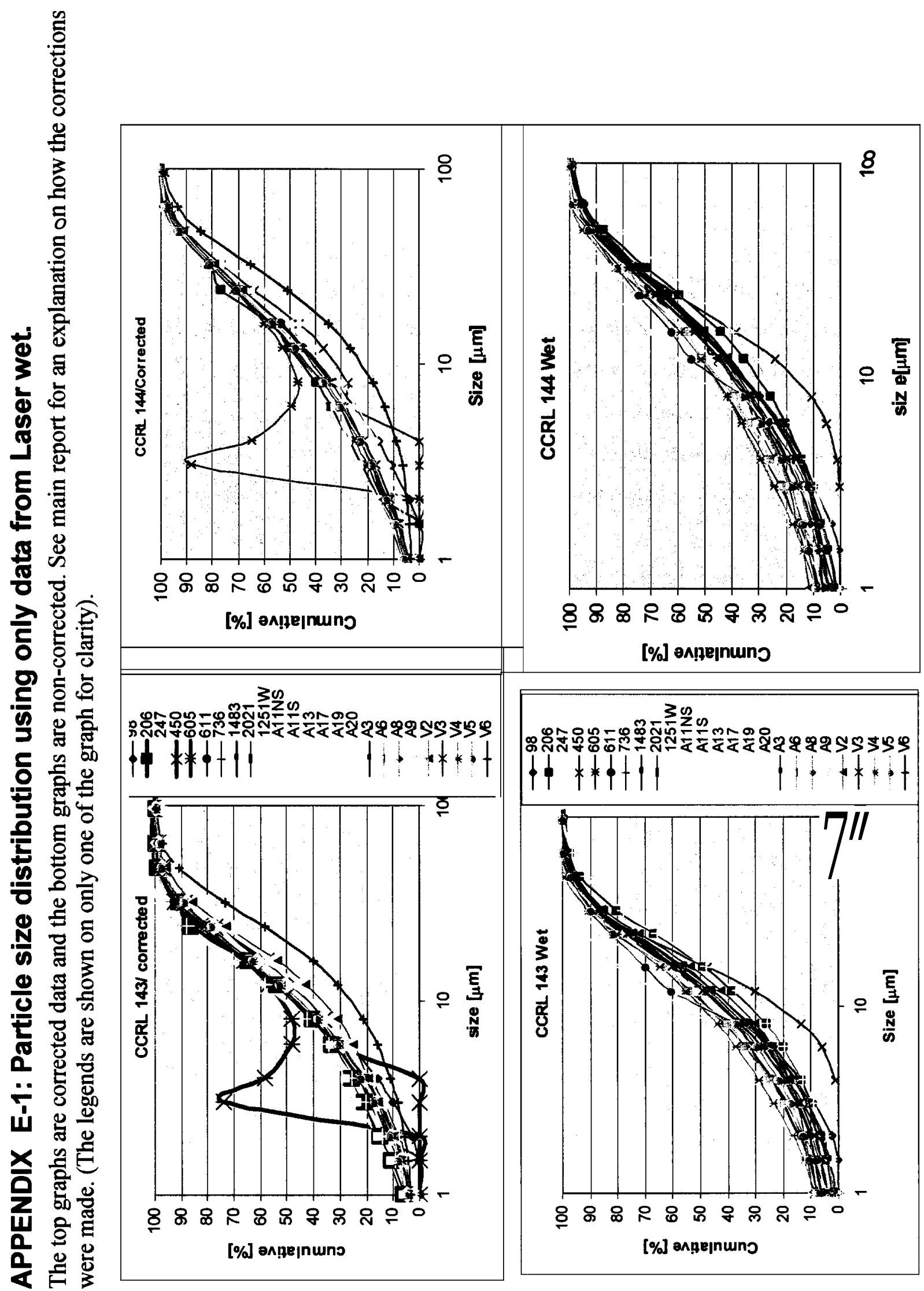




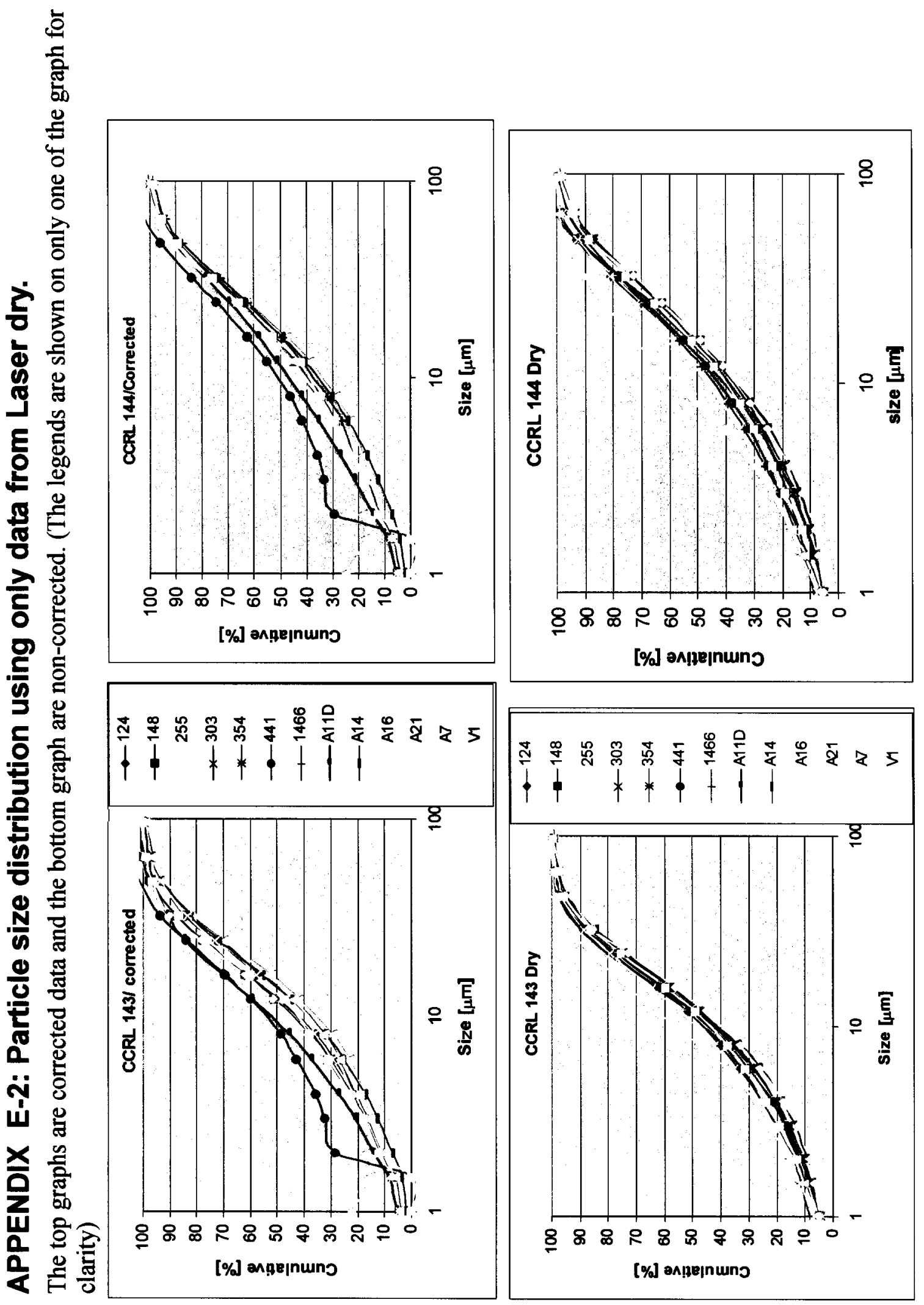


